\title{
The fine structure of the moduli space of abelian differentials in genus 3
}

\author{
Eduard Looijenga • Gabriele Mondello
}

Received: 22 September 2012 / Accepted: 25 February 2013

C) Springer Science+Business Media Dordrecht 2013

\begin{abstract}
The moduli space of curves endowed with a nonzero abelian differential admits a natural stratification according to the configuration of its zeroes. We give a description of these strata for genus 3 in terms of root system data. For each non-open stratum we obtain a presentation of its orbifold fundamental group.
\end{abstract}

Keywords Abelian differentials - Translation surfaces · Moduli space of curves · Root systems · Artin groups

Mathematics Subject Classification (2000) $\quad$ 14H45 $32 \mathrm{G} 15$

\section{Introduction}

The moduli space of pairs $(C, \varphi)$ with $C$ a complex connected smooth projective curve of genus $\mathrm{g} \geq 2$ and $\varphi$ a nonzero abelian differential on $\mathrm{C}$, and denoted here by $\mathcal{H}_{\mathrm{g}}$, comes with the structure of a Deligne-Mumford stack, but we will just regard it as an orbifold. The forgetful morphism $\mathcal{H}_{\mathrm{g}} \rightarrow \mathcal{M}_{\mathrm{g}}$ exhibits $\mathcal{H}_{\mathrm{g}}$ as the complement of the zero section of the Hodge bundle over $\mathcal{M}_{\mathrm{g}}$. A partition of $\mathcal{H}_{\mathrm{g}}$ into suborbifolds is defined by looking at the multiplicities of the zeros of the abelian differential: for any numerical partition $\mathbf{k}:=\left(\mathrm{k}_{1} \geq\right.$ $k_{2} \geq \cdots \geq k_{n}>0$ ) of $2 \mathrm{~g}-2$, we have a subvariety $\mathcal{H}^{\prime}(\mathbf{k}) \subset \mathcal{H}_{\mathrm{g}}$ which parameterizes the pairs $(C, \varphi)$ for which the zero divisor $Z_{\varphi}$ of $\varphi$ is of type $\mathbf{k}$. We call a stratum of $\mathcal{H}_{\mathrm{g}}$ a connected component of some $\mathcal{H}^{\prime}(\mathbf{k})$. (NB: our terminology slightly differs from that of [6]).

E. Looijenga

Mathematisch Instituut, Universiteit Utrecht, P.O. Box 80.010, 3508 TA Utrecht, The Netherlands

e-mail: E.J.N.Looijenga@uu.nl

G. Mondello $(\varangle)$

Dipartimento di Matematica "Guido Castelnuovo", "Sapienza" Università di Roma,

Piazzale Aldo Moro 5, 00185 Rome, Italy

e-mail: mondello@mat.uniroma1.it 


\subsection{Classification of strata}

Kontsevich and Zorich [6] characterized the strata of $\mathcal{H}_{\mathrm{g}}$ in rather simple terms. First consider the case when $C$ is hyperelliptic. Then an effective divisor of degree $2 g-2$ on $C$ is canonical if and only if it is invariant under the hyperelliptic involution. So the type $\mathbf{k}$ of such a divisor has the property that any odd integer appears in it an even number of times. There are two cases where the support of the canonical divisor is an orbit of the hyperelliptic involution: one is of type $(2 g-2)$ and the other is of type $(g-1, g-1)$ (the two cases corresponding to a Weierstraß point resp. a pair of points). The authors show that these make up strata and denote them by $\mathcal{H}^{\text {hyp }}(2 g-2)$ and $\mathcal{H}^{\text {hyp }}(g-1, g-1)$ respectively. Notice that this fully covers the case $\mathrm{g}=2$. They show that for $\mathrm{g} \geq 3$,

$$
\mathcal{H}(\mathbf{k}):= \begin{cases}\mathcal{H}^{\prime}(\mathbf{k})-\mathcal{H}^{\text {hyp }}(\mathbf{k}) & \text { for } \mathbf{k}=(2 \mathrm{~g}-2),(\mathrm{g}-1, \mathrm{~g}-1) \\ \mathcal{H}^{\prime}(\mathbf{k}) & \text { otherwise }\end{cases}
$$

is a stratum unless $\mathrm{g}>3$ and all the terms of $\mathbf{k}$ are even. In that case the canonical divisor is twice the divisor of an (effective) theta characteristic, which for $\mathrm{g}>3$ can be even or odd (for $\mathrm{g}=3$ it is necessarily odd, as there is no effective even theta characteristic). These loci are connected and hence define strata: $\mathcal{H}^{\text {even }}(\mathbf{k})$ and $\mathcal{H}^{\text {odd }}(\mathbf{k})$. This completes the KontsevichZorich characterization of the strata.

The possible codimension 1 degenerations within each stratum were analyzed by EskinMasur-Zorich in [3].

\subsection{Local structure of strata}

Each stratum is known to have a 'linear' structure: it comes with an atlas of holomorphic charts whose transition maps lie in $\operatorname{GL}(d, \mathbb{C})$, where $d$ is the dimension of the stratum. A chart of this atlas at $(C, \varphi)$ is defined as follows. Note that $\varphi$ defines an element $[\varphi] \in H^{1}\left(C, Z_{\varphi} ; \mathbb{C}\right)$, where $Z_{\varphi}$ denotes the zero locus consisting of $n$ distinct points. If we put $d:=2 g+\left|Z_{\varphi}\right|-1=$ $\operatorname{dim} H^{1}\left(C, Z_{\varphi} ; \mathbb{C}\right)$ and choose an isomorphism $H^{1}\left(C, Z_{\varphi} ; \mathbb{C}\right) \cong \mathbb{C}^{d}$, then varying $(C, \varphi)$ in a small neighborhood in its stratum makes the image of $[\varphi]$ vary in $\mathbb{C}^{\mathrm{d}}$. This yields a $\mathbb{C}^{\mathrm{d}}$-valued chart. In particular $\operatorname{dim} \mathcal{H}(\mathbf{k})=2 \mathrm{~g}+\mathrm{n}-1$.

\subsection{Projective classes of abelian differentials}

Since every connected stratum $\mathcal{S}$ of $\mathcal{H}_{\mathrm{g}}$ is invariant under scalar multiplication it defines a suborbifold $\mathbb{P S}$ in the $\mathbb{P}^{\mathrm{g}-1}$-orbifold bundle $\mathbb{P} \mathcal{H}_{\mathrm{g}}$ over $\mathcal{M}_{\mathrm{g}}$. Such a projectivized stratum parameterizes pairs $(C, D)$ with $C$ a smooth projective curve and $D$ a positive canonical divisor on $C$ with prescribed multiplicities. Moreover, $\mathcal{S}$ has a contractible orbifold universal cover if and only if $\mathbb{P S}$ has: in this case, the orbifold fundamental group of $\mathbb{P S}$ is the quotient of the orbifold fundamental group of $\mathcal{S}$ by an infinite cyclic central subgroup. The somewhat technical price to pay for working with $\mathbb{P S}$ is that over the hyperelliptic locus we have to deal with $\mathbb{Z} / 2$-gerbes.

Since $\mathcal{S}$ has a linear structure, $\mathbb{P} \mathcal{S}$ has a projective structure (i.e., it has a holomorphic atlas which takes values in $\mathbb{P}^{\operatorname{dim} \mathcal{S}-1}$ such that the transition maps lie in a projective linear group). 


\subsection{Hyperelliptic strata}

The topology of the hyperelliptic strata is familiar and although a bit subtle, essentially well understood. Consider an affine plane curve $\mathrm{C}_{\rho}^{\circ}$ with equation

$$
w^{2}=\prod_{i=1}^{2 g+1}\left(z-\rho_{i}\right)
$$

where the $\rho_{i}$ 's are pairwise distinct. A projective curve $C_{\rho}$ is obtained by adding a point $p$ at infinity and $w^{-1} \mathrm{~d} z$ extends over $C_{\rho}$ as an abelian differential $\varphi_{\rho}$ with a unique zero at infinity. Notice that $\varphi_{\rho}$ naturally defines a nonzero element in $\left(T_{p}^{*} C_{\rho}\right)^{\otimes(2 g-1)}$.

Performing the above construction in families over

$$
\mathcal{R}_{2 g+1}:=\left\{\rho \in \mathbb{C}^{2 g+1} \mid \sum_{i} \rho_{i}=0, \rho_{i} \neq \rho_{j}\right\}
$$

we obtain a diagram

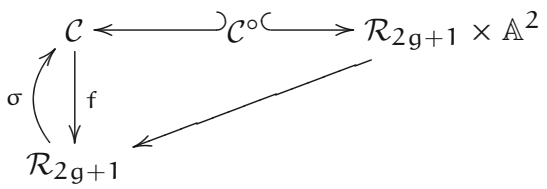

where $f$ is a smooth projective curve with a section $\sigma$ representing the point at infinity and $\mathcal{C}^{\circ}$ is the complement of the divisor $\mathcal{D}=\sigma\left(\mathcal{R}_{2 \mathrm{~g}+1}\right)$ inside $\mathcal{C}$. Moreover, we also obtain a $\Phi \in \mathrm{H}^{0}\left(\mathcal{C}, \omega_{f}\right)$ that vanishes along the image of $\sigma$ of order $2 \mathrm{~g}-2$ and so a nowhere-zero section of $\left(\sigma^{*} \omega_{f}\right)^{\otimes(2 g-1)}$ over $\mathcal{R}_{2 g+1}$.

The $\mathbb{G}_{\mathrm{m}}$-action on $\mathcal{R}_{2 \mathrm{~g}+1}$ defined as $\zeta \cdot \rho:=\zeta^{2} \rho$ lifts on $\mathcal{R}_{2 \mathrm{~g}+1} \times \mathbb{A}^{2}$ as $\zeta \cdot(\rho, z, w):=$ $\left(\zeta^{2} \rho, \zeta^{2} z, \zeta^{2 g+1} w\right)$ preserving $\mathcal{C}^{\circ}$ and $\sigma$. Notice that $-1 \in \mathbb{G}_{\mathrm{m}}$ yields the hyperelliptic involution. The induced action on $\Phi$ is $\zeta \cdot \varphi_{\rho}:=\zeta^{1-2 \mathrm{~g}} \varphi_{\zeta^{2} \rho}$, and so $\left(\mathrm{C}_{\rho}, \varphi_{\rho}\right)$ is isomorphic to $\left(C_{\rho^{\prime}}, \varphi_{\rho^{\prime}}\right)$ if and only if $\rho^{\prime}=\zeta \cdot \rho$ with $\zeta \in \mu_{2 g-1}$. Moreover, the action of $\mathfrak{S}_{2 g+1}$ on $\mathcal{R}_{2 \mathrm{~g}+1}$ that permutes the components of $\rho$ commutes with the $\mathbb{G}_{m}$-action and so we obtain an isomorphism of orbifolds

$$
\mathcal{R}_{2 \mathrm{~g}+1} /\left(\mathfrak{S}_{2 \mathrm{~g}+1} \times \mu_{2 \mathrm{~g}-1}\right) \longrightarrow \mathcal{H}^{\text {hyp }}(2 \mathrm{~g}-2)
$$

Now, $\mathcal{R}_{2 g+1} / \mathfrak{S}_{2 g+1}$ is a classifying space for the braid group $\mathrm{B}_{2 \mathrm{~g}+1}$ on $2 \mathrm{~g}+1$ strands. Hence $\mathcal{H}^{\text {hyp }}(2 g-2)$ is an orbifold classifying space for a group that is an extension of $\mu_{2 g-1}$ by $B_{2 g+1}$. If we are interested in projective classes of abelian differentials, then we obtain

$$
\mathcal{R}_{2 \mathrm{~g}+1} /\left(\mathfrak{S}_{2 \mathrm{~g}+1} \times \mathbb{G}_{\mathrm{m}}\right) \longrightarrow \mathbb{P H}^{\mathrm{hyp}}(2 \mathrm{~g}-2)
$$

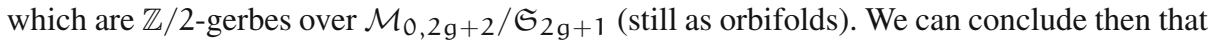
$\mathbb{P} \mathcal{H}^{\text {hyp }}(2 \mathrm{~g}-2)$ has a contractible orbifold universal cover and its orbifold fundamental group is a hyperelliptic mapping class group, namely the centralizer in the mapping class group of a hyperelliptic involution $\tau$ of a pointed genus $g$ surface (which preserves the point).

Similarly for $\mathcal{H}^{\text {hyp }}(\mathrm{g}-1, \mathrm{~g}-1)$, we consider affine plane curves $\mathrm{C}_{\rho}^{\circ}$ of equation

$$
w^{2}=\prod_{i=1}^{2 g+2}\left(z-\rho_{i}\right)
$$


together with the differential $\varphi_{\rho}=w^{-1} \mathrm{~d} z$. If $C_{\rho}$ is the smooth completion of $C_{\rho}^{\circ}$ obtained by adding the points at infinity $p_{1}$ and $p_{2}$, then $\varphi_{\rho}$ naturally determines a nonzero element in $\left(\mathrm{T}_{\mathrm{p}_{1}}^{*} \mathrm{C}_{\rho} \otimes \mathrm{T}_{\mathrm{p}_{2}}^{*} \mathrm{C}_{\rho}\right)^{\otimes \mathrm{g}}$.

Implementing the above construction in families yields a smooth curve $f: \mathcal{C} \rightarrow \mathcal{R}_{2 \mathrm{~g}+2}=$ $\left\{\rho \in \mathbb{C}^{2 g+2} \mid \sum_{i} \rho_{i}=0, \rho_{i} \neq \rho_{j}\right\}$ and a $\Phi \in H^{0}\left(\mathcal{C}, \omega_{f}\right)$ whose divisor is of the form $(\mathrm{g}-1) \mathcal{D}$, where $\mathcal{D}$ is reduced and projects étale $2: 1$ onto $\mathcal{R}_{2 g}+2$. So $\Phi$ defines a nowherezero section of $\operatorname{det}\left(f_{*}\left(\omega_{f} \otimes \mathcal{O}_{\mathcal{D}}\right)\right)^{\otimes g}$. A $\mathbb{G}_{m}$-action can be defined on $\mathcal{R}_{2 \mathrm{~g}+2} \times \mathbb{A}^{2}$ as $\zeta \cdot(\rho, z, w):=\left(\zeta \rho, \zeta z, \zeta^{\mathrm{g}+1} w\right)$. Observe that $\zeta \cdot \Phi:=\zeta^{-\mathrm{g}} \Phi$, or equivalently, that under the isomorphism $\zeta: C_{\rho} \stackrel{\sim}{\longrightarrow} C_{\zeta \rho}$, the pull-back of $\varphi_{\zeta \rho}$ is $\zeta^{-g} \varphi_{\rho}$. We also have the obvious action of $\mathfrak{S}_{2 \mathrm{~g}+2}$ on this family (which just permutes the $\rho_{i}$ 's) and the involution $\tau$ which sends $(\rho, z, w)$ to $(\rho, z,-w)$, and so $\tau \cdot \varphi_{\rho}=-\varphi_{\rho}$. These three actions commute, so that we have one of $\mathfrak{S}_{2 g+2} \times \mathfrak{S}_{2} \times \mathbb{G}_{\mathfrak{m}}$. The stabilizer of $\Phi$ is $\mathfrak{S}_{2 \mathrm{~g}+2} \times \mu_{\mathrm{g}}$ and it is easy to see that any isomorphism $\left(C_{\rho}, \varphi_{\rho}\right) \cong\left(C_{\rho^{\prime}}, \varphi_{\rho^{\prime}}\right)$ is the restriction of an element of this stabilizer. So we obtain the isomorphism of orbifolds

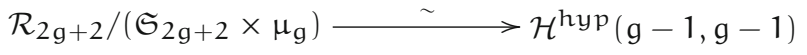

which exhibits $\mathcal{H}^{\text {hyp }}(\mathrm{g}-1, \mathrm{~g}-1)$ as an orbifold classifying space of an extension of $\mu_{\mathrm{g}}$ by

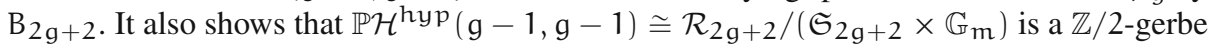
over $\mathcal{M}_{0,2 g+3} / \mathfrak{S}_{2 g+2}$ and therefore its orbifold universal cover is contractible. Moreover, the orbifold fundamental group of $\mathbb{P} \mathcal{H}^{\text {hyp }}(g-1, g-1)$ is just a hyperelliptic mapping class group: to be precise, it is the centralizer in the full mapping class group of a hyperelliptic involution of a twice pointed genus $g$ surface which exchanges the points.

In their preprint [5] Kontsevich and Zorich conjecture that something similar is true in general, namely that each projectivized stratum always has a contractible orbifold universal cover and that its orbifold fundamental group is commensurable with some mapping class group.

\subsection{The other strata in genus 3}

In this paper we give rather precise descriptions of all the strata in genus 3. This enables us to find a presentation of their orbifold fundamental group, at least in principle: we do this for all the strata, except for the open stratum $\mathcal{H}\left(1^{4}\right)$, where it gets unwieldy, and to make for these strata the Kontsevich-Zorich conjecture so explicit that it acquires more of a topological flavor. Concretely, we show that the nonhyperelliptic strata in genus 3 can be understood as parameterizing del Pezzo surfaces of degree two or one endowed with an anticanonical divisor of a given type and describe these in turn in terms of combinatorial (root) data. Such moduli spaces have been investigated by one of us before. It turns out that they can be given in a somewhat similar spirit as the hyperelliptic strata: some orbifold cover appears as the complement of a locally finite arrangement in a domain and the in principle their fundamental group can be computed. For instance, for $\mathcal{H}(3,1)$ resp. $\mathcal{H}(4)$ we get the discriminant complement of the root system of type $\mathbf{E}_{7}$ resp. $\mathbf{E}_{6}$. Its fundamental group is the Artin group of that type and a highly nontrivial theorem of Deligne [2] asserts that this complement has indeed a contractible universal cover. But in the other cases this seems difficult to establish. Questions of that kind are reminiscent, and indeed overlap, with the Arnol'd-Thom conjecture which states that the discriminant complement of the universal deformation of an an isolated hypersurface singularity is a $K(\Gamma, 1)$. They are still the subject of current research [1]. 
We do not know how to make the commensurability conjecture of Kontsevich and Zorich precise. Our results seem to indicate that the open stratum $\mathcal{H}\left(1^{4}\right)$ has an orbifold fundamental group which may not be commensurable with a central extension of the mapping class group of the punctured genus 3 curve. In fact, for none of the strata described here, we were able to characterize their orbifold fundamental group as a kind of a mapping class group.

\section{Genus 3 strata in terms of del Pezzo surfaces}

Let $C$ be a nonhyperelliptic nonsingular curve of genus 3 . The canonical system on $C$ embeds in a projective plane $P_{C}$. A double cover $\pi: X_{C} \rightarrow P_{C}$ of this plane which totally ramifies over $C$ is unique up to the obvious involution. The covering variety $X_{C}$ is a del Pezzo surface of degree 2, which here amounts to saying that the anticanonical system on $X_{C}$ is ample and is given by the morphism $\pi$ : any effective anticanonical divisor on $X_{C}$ is the pull-back of a line in $\mathrm{P}_{\mathrm{C}}$.

We are now going to recall some known results from [7], Section 1.

\subsection{Brief review of degree two del Pezzo surfaces}

Every effective anticanonical divisor on $X_{C}$ has arithmetic genus one (this is also clear from the way we defined $X_{C}$ ). If $L \subset P_{C}$ is a double tangent of $C$, then its preimage in $X_{C}$ consists of two exceptional curves (an exceptional curve is a smooth genus zero curve with selfintersection -1) which intersect each other with intersection number 2. Since there are 28 bitangents we get $2 \cdot 28=56$ exceptional curves and these are in fact all of them. If we select 7 such exceptional curves $E_{1}, E_{2}, \ldots, E_{7}$ that are pairwise disjoint (geometric marking), then their contraction yields a map $X_{C} \rightarrow \mathbb{P}^{2}$ and the anticanonical system is then realized as the system of cubics passing through the 7 image points of this $\mathbb{P}^{2}$. There are however many ways of picking 7 pairwise disjoint copies and the best way to come to terms with this is to invoke an associated symmetry group, which is a Weyl group of type $\mathbf{E}_{7}$.

Let us make this precise. The natural map $\operatorname{Pic}\left(X_{C}\right) \rightarrow H^{2}\left(X_{C}\right)$ is an isomorphism. The latter is free of rank 8 and $\left\{\ell, e_{1}, e_{2}, \ldots, e_{7}\right\}$ is a basis, where $e_{1}, \ldots, e_{7}$ are the classes of the exceptional curves $E_{1}, \ldots, E_{7}$ mentioned above and $\ell$ is the pull-back of the class of a line on $\mathbb{P}^{2}$. One can easily check that the intersection pairing is diagonal with respect to such a basis and it has signature $(1,7)$, because $\ell \cdot \ell=1, \ell \cdot e_{i}=0$ and $e_{i} \cdot e_{j}=-\delta_{i j}$. If $-K=3 \ell-\left(e_{1}+\cdots+e_{7}\right) \in \operatorname{Pic}\left(X_{C}\right)$ stands for the anticanonical class, then $(-K)^{2}=2$. So the orthogonal complement of $-\mathrm{K}$ in $\mathrm{Pic}\left(\mathrm{X}_{\mathrm{C}}\right)$, denoted here by $\mathrm{Pic}_{\circ}\left(\mathrm{X}_{\mathrm{C}}\right)$, is negative definite and a basis is given by $\left\{\ell-\left(e_{1}+e_{2}+e_{3}\right), e_{1}-e_{2}, e_{2}-e_{3}, \ldots, e_{6}-e_{7}\right\}$. Note that the intersection matrix of these elements is minus the Cartan matrix of an $\mathbf{E}_{7}$ root system.

A root is an element $\alpha \in \mathrm{Pic}_{\circ}\left(\mathrm{X}_{\mathrm{C}}\right)$ with $\alpha \cdot \alpha=-2$. Associated to a root $\alpha$ is a reflection $s_{\alpha}$ in $\operatorname{Pic}\left(X_{C}\right)$, given by $u \in \operatorname{Pic}\left(X_{C}\right) \mapsto u+(u \cdot \alpha) \alpha$, which fixes $-K$ and preserves the intersection pairing. These reflections generate a Weyl group $W\left(X_{C}\right)$ of type $\mathbf{E}_{7}$ so that the roots make up a root system $\mathfrak{R}\left(\mathrm{X}_{\mathrm{C}}\right)$ of the same type.

It is known that the classes of the exceptional curves generate $\operatorname{Pic}\left(X_{C}\right)$ and that the roots generate $\mathrm{Pic}_{\circ}\left(\mathrm{X}_{\mathrm{C}}\right)$. A root can be represented by the difference of two disjoint exceptional curves, although not uniquely so. The Weyl group $W\left(X_{C}\right)$ is the full stabilizer of $-K$ in the orthogonal group of $\operatorname{Pic}\left(X_{C}\right)$. The involution of $X_{C}$ preserves $-K$ and acts as minus the identity in $\operatorname{Pic}_{\circ}\left(X_{C}\right)$, hence appears here as the central element of $W\left(X_{C}\right)$. But the other nontrivial elements of $W\left(X_{C}\right)$ are usually not induced by an automorphism of $X_{C}$. 
Denote by $\mathrm{Q}\left(\mathbf{E}_{7}\right)$ a fixed root lattice of type $-\mathbf{E}_{7}$. A marking of $X_{C}$ is an isometry between $\mathrm{Pic}_{\circ}\left(\mathrm{X}_{\mathrm{C}}\right)$ and $\mathrm{Q}\left(\mathbf{E}_{7}\right)$. It is well-known (Manin) that all such markings are geometric, i.e. they are determined as above by a collection of 7 disjoint exceptional curves, and that $W\left(\mathbf{E}_{7}\right)$ acts simply and transitively on the markings.

\subsection{Bringing in a canonical divisor}

Let $\mathrm{D}$ be a positive canonical divisor on $\mathrm{C}$. Then $\mathrm{D}$ is the trace of a line $\mathrm{L}_{\mathrm{D}} \subset \mathrm{P}_{\mathrm{C}}$ on $\mathrm{C}$ and $\tilde{L}_{D}:=\pi^{*} L_{D}$ is an anticanonical divisor on $X_{C}$. As we noted, the latter is of arithmetic genus one: if $D$ is general (of type $(1,1,1,1)$ ), then $\tilde{L}_{D}$ is smooth and in the other cases $\tilde{L}_{D}$
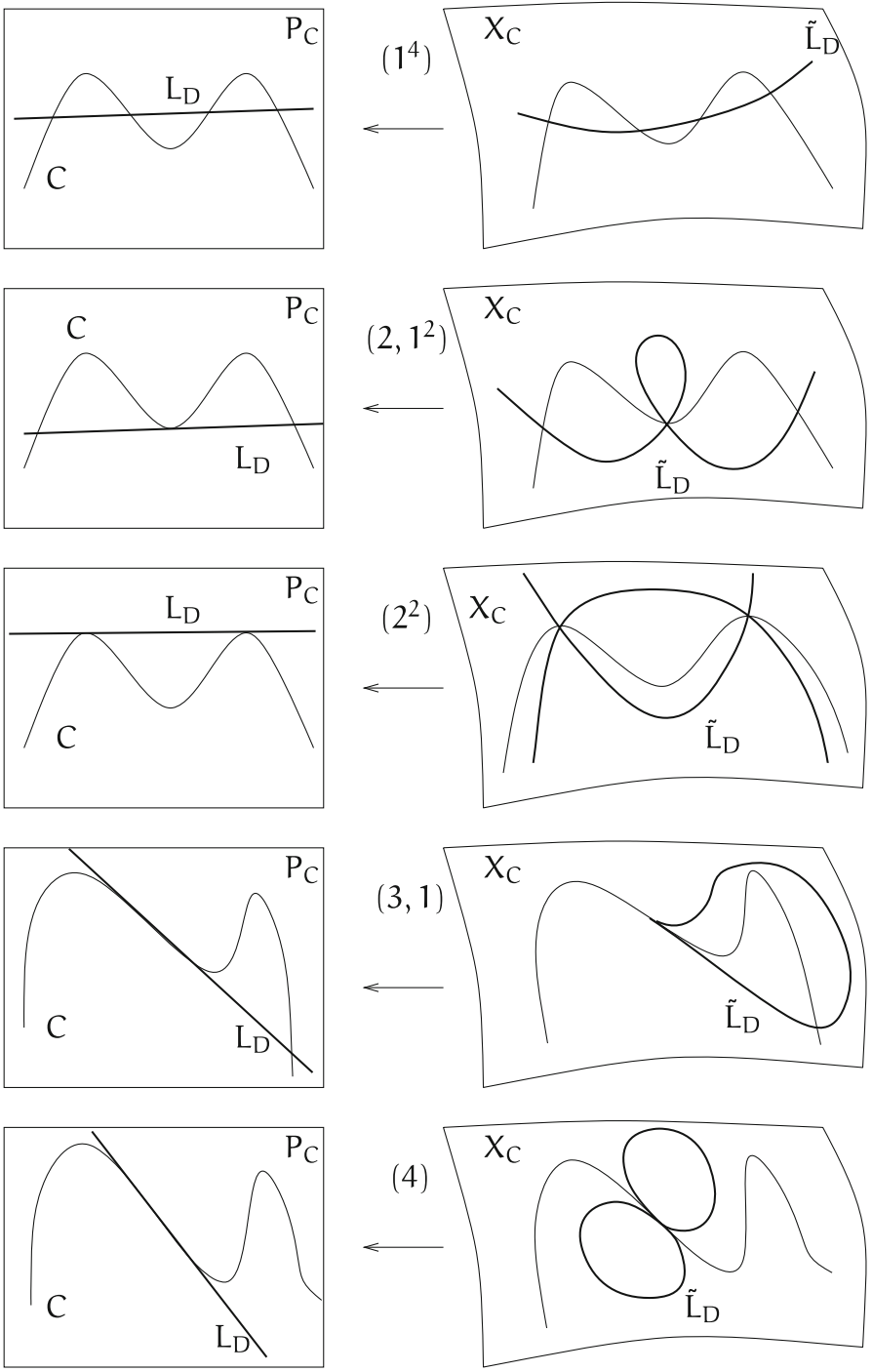

Fig. 1 The non-hyperelliptic strata and the cover $X_{C} \rightarrow P_{C}$ 
Table 1 Nonhyperelliptic strata in genus 3

\begin{tabular}{lll}
\hline $\mathbf{k}$ & Kodaira type & Type of $\Re\left(\mathrm{X}_{\mathrm{C}}, \tilde{\mathrm{L}}_{\mathrm{D}}\right)$ \\
\hline$\left(1^{4}\right)$ & Smooth & $\mathbf{E}_{7}$ \\
$\left(2,1^{2}\right)$ & $\mathrm{I}_{1}$ (mult) & $\mathbf{E}_{7}$ \\
$\left(2^{2}\right)$ & $\mathrm{I}_{2}$ (mult) & $\mathbf{E}_{6}$ \\
$(3,1)$ & II (add) & $\mathbf{E}_{7}$ \\
$(4)$ & III (add) & $\mathbf{E}_{6}$ \\
\hline
\end{tabular}

is a nodal curve ( $D$ is of type $(2,1,1)$ ), a bigon (type $I_{2}$ in the Kodaira classification and $\mathrm{D}$ is of type $(2,2)$ ), a cuspidal curve (type II in the Kodaira classification and D is of type $(3,1)$ ) or two smooth rational curves meeting in a point with multiplicity 2 (type III in the Kodaira classification and D is of type (4)). We regard $\tilde{L}_{D}$ as a genus one curve endowed with a polarization of degree 2 (Fig. 1).

The group $\operatorname{Pic}\left(\tilde{L}_{D}\right)$ of isomorphism classes of line bundles on $\tilde{L}_{D}$ has as its identity component $\operatorname{Pic}^{0}\left(\tilde{\mathrm{L}}_{\mathrm{D}}\right)$ an elliptic curve, is isomorphic to $\mathbb{C}^{\times}$or is the additive group $\mathbb{C}$, according to whether $\mathrm{D}$ is reduced, has a point of multiplicity 2 , or has a point of multiplicity $\geq 3$.

The orthogonal complement in $\operatorname{Pic}\left(X_{C}\right)$ of the classes of the irreducible components of $\tilde{\mathrm{L}}_{\mathrm{D}}$, denoted here by $\operatorname{Pic}\left(\mathrm{X}_{C}, \tilde{\mathrm{L}}_{\mathrm{D}}\right) \subset \operatorname{Pic}\left(\mathrm{X}_{C}\right)$, is generated by the roots contained in it, so that these roots make up a root subsystem $\mathfrak{R}\left(\mathrm{X}_{C}, \tilde{\mathrm{L}}_{D}\right) \subset \mathfrak{R}\left(\mathrm{X}_{C}\right)$. We have only two cases: for $\tilde{L}_{D}$ irreducible, we have of course $\mathfrak{R}\left(\mathrm{X}_{C}, \tilde{\mathrm{L}}_{\mathrm{D}}\right)=\mathfrak{R}\left(\mathrm{X}_{C}\right)$ and otherwise (when $\tilde{\mathrm{L}}_{\mathrm{D}}$ has two irreducible components interchanged by $\iota), \mathfrak{R}\left(X_{C}, \tilde{L}_{D}\right)$ is of type $\mathbf{E}_{6}$ (see Table 1).

\subsection{The basic invariant}

The natural homomorphism $\operatorname{Pic}\left(X_{C}\right) \rightarrow \operatorname{Pic}\left(\tilde{L}_{D}\right)$ has an evident restriction

$$
\chi_{C, D}: \operatorname{Pic}\left(X_{C}, \tilde{\mathrm{L}}_{D}\right) \rightarrow \operatorname{Pic}^{0}\left(\tilde{\mathrm{L}}_{D}\right) .
$$

It plays a central role in what follows. Let us first observe that no root $\alpha \in \Re\left(X_{C}, \tilde{L}_{D}\right)$ lies in the kernel of $\chi_{C, D}$. For such a root $\alpha$ can be represented by a difference $E-E^{\prime}$ of disjoint exceptional curves $E, E^{\prime}$ which meet the same component of $\tilde{L}_{D}$, reg, in $p$ resp. $p^{\prime}$, say. Then clearly, $(p)-\left(p^{\prime}\right)$ represents $\chi_{C, D}(\alpha)$ and since $p \neq p^{\prime}$, it is a nonzero element of $\operatorname{Pic}^{0}\left(\tilde{L}_{D}\right)$.

We now view $\chi_{C, D}$ as an element of $\operatorname{Pic}\left(X_{C}, \tilde{L}_{D}\right)^{\vee} \otimes \operatorname{Pic}^{0}\left(\tilde{L}_{D}\right)$. As shown in [7] (Section 1), this last group is a weight lattice of type $\mathbf{E}_{6}$ or $\mathbf{E}_{7}$ tensored with either an elliptic curve, a copy of $\mathbb{C}^{\times}$or a copy of $\mathbb{C}$ (which is like a six- or sevenfold power of the latter but with a Weyl group symmetry built in); moreover, its isomorphism type is a complete invariant of the pair $\left(X_{C}, \tilde{L}_{D}\right)$ and hence of the pair $(C, D)$. To be more concrete, let us identify $\mathfrak{R}(C, D)$ with a fixed root system $\Re$ of type $\mathbf{E}_{6}$ or $\mathbf{E}_{7}$. Two such identifications differ by an element of the automorphism group $\operatorname{Aut}(\mathfrak{R})$ of $\Re$, which is $\{ \pm 1\}$.W $(\Re)$ in the $\mathbf{E}_{6}$ case and $W(\Re)$ in the $\mathbf{E}_{7}$ case. We also identify $\operatorname{Pic}^{0}\left(\tilde{L}_{D}\right)$ with a fixed group $G$, which is either an elliptic curve, or the multiplicative group $\mathbb{C}^{\times}$or the additive group $\mathbb{C}$. Two such identifications differ by an automorphism of $G$. Notice that $\operatorname{Aut}(G)$ equals $\{ \pm 1\}$ for $G$ a generic elliptic curve or $G \cong \mathbb{C}^{\times}$and is equal to $\mathbb{C}^{\times}$when $G \cong \mathbb{C}$. As the involution $\iota$ of $X_{C}$ acts as -1 both on $\Re$ and on $G$, the choices of a marking and of an identification $\operatorname{Pic}^{0}\left(\tilde{L}_{D}\right) \cong G$ are permuted by $\operatorname{Aut}(\Re, G):=\operatorname{Aut}(\Re) \times \operatorname{Aut}(G) /(-1,-1)$. 
Hence, if we denote by $Q(\Re)$ the (root) lattice spanned by $\Re$, and by $\operatorname{Hom}(Q(\Re), G)^{\circ}$ the subset of homomorphisms with no root in their kernel, then our $\chi \mathrm{C}, \mathrm{D}$ defines an element of

$$
\mathrm{S}(\Re, \mathrm{G}):=\operatorname{Aut}(\Re, \mathrm{G}) \backslash \operatorname{Hom}(\mathrm{Q}(\mathfrak{R}), \mathrm{G})^{\circ}
$$

for $\Re$ and $\mathrm{G}$ as listed. This construction also makes sense for families of elliptic curves. In fact, if $\mathcal{C}_{1,1} / \mathcal{M}_{1,1}$ is the universal elliptic curve (an orbifold), then we can form $S\left(\Re, \mathcal{C}_{1,1} / \mathcal{M}_{1,1}\right)$. Let us write $\mathcal{M}_{0,(4)}$ for the moduli stack of 4-element subsets of $\mathbb{P}^{1}$ up to projective equivalence, in other words, $\mathcal{M}_{0,(4)}:=\mathfrak{S}_{4} \backslash \mathcal{M}_{0,4}$. There is an evident $\mathbb{Z} / 2$-gerbe $\mathcal{M}_{1,1} \rightarrow \mathcal{M}_{0,(4)}$ (a 'B(Z/Z)-fibration'), that makes $S\left(\Re, \mathcal{C}_{1,1} / \mathcal{M}_{1,1}\right)$ fiber over $\mathcal{M}_{0,(4)}$.

Theorem 2.1 The map (C, D) $\mapsto \chi_{\mathrm{C}, \mathrm{D}}$ induces orbifold isomorphisms

$$
\begin{aligned}
\mathbb{P} \mathcal{H}(4) & \cong \mathrm{S}\left(\mathbf{E}_{6}, \mathbb{C}\right), \\
\mathbb{P} \mathcal{H}(3,1) & \cong \mathrm{S}\left(\mathbf{E}_{7}, \mathbb{C}\right), \\
\mathbb{P} \mathcal{H}\left(2^{2}\right)-\mathbb{P} \mathcal{H}\left(2^{2}\right)_{\text {hyp }} & \cong \mathrm{S}\left(\mathbf{E}_{6}, \mathbb{C}^{\times}\right), \\
\mathbb{P} \mathcal{H}\left(2,1^{2}\right)-\mathbb{P} \mathcal{H}\left(2,1^{2}\right)_{\text {hyp }} & \cong \mathrm{S}\left(\mathbf{E}_{7}, \mathbb{C}^{\times}\right),
\end{aligned}
$$

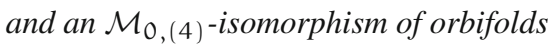

$$
\mathbb{P H}\left(1^{4}\right)-\mathbb{P} \mathcal{H}\left(1^{4}\right)_{\text {hyp }} \cong \mathrm{S}\left(\mathbf{E}_{7}, \mathcal{C}_{1,1} / \mathcal{M}_{1,1}\right) .
$$

Here $\mathbb{P} \mathcal{H}(\mathbf{k})_{\text {hyp }} \subset \mathbb{P H}(\mathbf{k})$ denotes the locus in $\mathbb{P} \mathcal{H}(\mathbf{k})$ for which the underlying curve is hyperelliptic.

Proof The result for the non-open strata follows from the above discussion and from [7], Section 1. Mimicking the strategy followed in that paper, we are going to reverse the above construction for the open stratum.

Consider the lattice freely generated by $\left\{e_{1}, \ldots, e_{7}, \ell\right\}$ and endowed with the intersection pairing $\ell \cdot \ell=1, \ell \cdot e_{i}=0, e_{i} \cdot e_{j}=-\delta_{i j}$ and fix an isometry between $Q\left(\mathbf{E}_{7}\right)$ and the subspace orthogonal to $3 \ell-\left(e_{1}+\cdots+e_{7}\right)$, as seen at the beginning of the section.

A point $\chi \in \operatorname{Hom}\left(\mathrm{Q}\left(\mathbf{E}_{7}\right), \mathcal{C}_{1,1} / \mathcal{M}_{1,1}\right)^{\circ}$ determines an elliptic curve $(\tilde{\mathrm{L}}, \mathrm{p}) \in \mathcal{M}_{1,1}$ and a homomorphism $\chi: Q\left(\mathbf{E}_{7}\right) \rightarrow \operatorname{Pic}^{0}(\tilde{\mathrm{L}})$. Define the points $\mathrm{p}_{1}, \ldots, \mathrm{p}_{7}$ on $\tilde{\mathrm{L}}$ as $\mathrm{p}_{1}:=\mathrm{p}$ and by $\mathcal{O}_{\tilde{\mathrm{L}}}\left(p_{i+1}\right):=\mathcal{O}_{\tilde{\mathrm{L}}}\left(p_{i}\right) \otimes \chi\left(e_{i+1}-e_{i}\right)$. Then the linear system $\mathcal{O}_{\tilde{\mathrm{L}}}\left(p_{1}+p_{2}+p_{3}\right) \otimes \chi(\ell-$ $\left.e_{1}-e_{2}-e_{3}\right)$ of degree 3 provides an embedding of $\tilde{L}$ in a projective plane.

The condition that $\chi(\alpha) \neq 1$ for all roots $\alpha \in \mathrm{Q}\left(\mathbf{E}_{7}\right)$ ensures that the image of the points $\mathrm{P}_{1}, \ldots, \mathrm{P}_{7}$ are in general position in the projective plane. Blowing them up, we obtain a del Pezzo surface of degree 2 and its anti-canonical system determines a smooth non-hyperelliptic genus 3 curve $C$ (its branch locus) and a line L (the image of $\tilde{\mathrm{L}}$ ).

As this construction is $W\left(\mathbf{E}_{7}\right)$-invariant and can be performed in families, we have then obtained the wished map $S\left(\mathbf{E}_{7}, \mathcal{C}_{1,1} / \mathcal{M}_{1,1}\right) \rightarrow \mathbb{P H}\left(1^{4}\right)-\mathbb{P H}\left(1^{4}\right)_{\text {hyp }}$.

The main result of Deligne in [2] implies that any variety of the form $S(\Re, \mathbb{C})$ is an orbifold classifying space of its orbifold fundamental group. If $\mathfrak{R}$ has the property that its automorphism group is $\{ \pm 1\}$. $W(\Re)$ or $W(\Re)$ (which is the case when it is of type $\mathbf{A}$ or $\mathbf{E}$ ), then the orbifold fundamental group in question equals the quotient of the Artin group of type $W(\Re)$ by its natural (infinite cyclic) central subgroup. Hence we find:

Corollary 2.2 The stratum $\mathbb{P H}(4)$ resp. $\mathbb{P H}(3,1)$ is an orbifold classifying space for the Artin group of type $\mathbf{E}_{7}$ resp. $\mathbf{E}_{6}$ modulo its natural (infinite cyclic) central subgroup.

But we do not know how to characterize any of these groups as a kind of a mapping class group. 


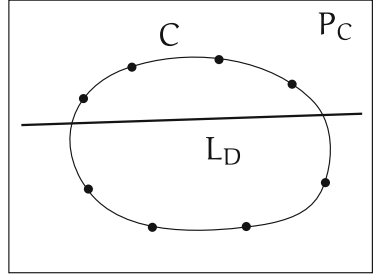

$\left(1^{4}\right)$

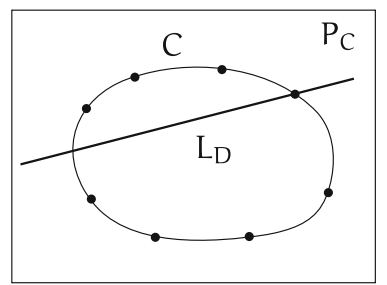

$\left(2,1^{2}\right)$

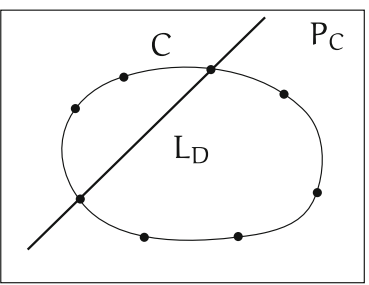

$\left(2^{2}\right)$

Fig. 2 Hyperelliptic degenerations in the strata $\left(1^{4}\right),\left(2,1^{2}\right),\left(2^{2}\right)$

\section{The hyperelliptic locus}

The strata corresponding to $\mathbf{k}=\left(1^{4}\right),\left(2,1^{2}\right),\left(2^{2}\right)$ contain both non-hyperelliptic and hyperelliptic curves. Thus, we must know how adding $\mathbb{P} \mathcal{H}(\mathbf{k})_{\text {hyp }}$ to $\mathbb{P} \mathcal{H}(\mathbf{k})-\mathbb{P} \mathcal{H}(\mathbf{k})_{\text {hyp }}$ can be expressed in terms of the right hand side in Theorem 2.1. Let us first give each of the $\mathbb{P} \mathcal{H}(\mathbf{k})_{\text {hyp }}$ a description in the same spirit as the varieties we dealt with (Fig. 2).

Let us first observe that the hyperelliptic involution gives each $\mathbb{P H}(\mathbf{k})_{\text {hyp }}$ the structure of a $\mathbb{Z} / 2$-gerbe. The following proposition identifies the base:

Proposition 3.1 The hyperelliptic involution yields natural $\mathbb{Z} / 2$-gerbes

$$
\begin{aligned}
\mathbb{P} \mathcal{H}\left(1^{4}\right)_{\text {hyp }} & \rightarrow \mathrm{S}\left(\mathbf{A}_{7}, \mathbb{C}^{\times}\right), \\
\mathbb{P H}\left(2,1^{2}\right)_{\text {hyp }} & \rightarrow \mathrm{W}\left(\mathbf{A}_{6}\right) \backslash \operatorname{Hom}\left(\mathrm{Q}\left(\mathbf{A}_{6}\right), \mathbb{C}^{\times}\right)^{\circ}, \\
\mathbb{P H}(2,2)_{\text {hyp }} & \rightarrow \mathrm{S}\left(\mathbf{A}_{5}, \mathbb{C}^{\times}\right),
\end{aligned}
$$

where we note that $\mathrm{W}\left(\mathbf{A}_{6}\right) \backslash \operatorname{Hom}\left(\mathrm{Q}\left(\mathbf{A}_{6}\right), \mathbb{C}^{\times}\right)^{\circ} \rightarrow \mathrm{S}\left(\mathbf{A}_{6}, \mathbb{C}^{\times}\right)$is a double cover.

Proof The loci in question are the moduli stacks for the pairs (C, D) for which $\mathrm{C}$ is a hyperelliptic genus three curve and $\operatorname{supp}(\mathrm{D})$ is the union of two distinct orbits under the hyperelliptic involution. The three cases correspond to having 0,1 , or 2 Weierstraß points in $\operatorname{supp}(\mathrm{D})$. If we divide out by the hyperelliptic involution, we get a copy of $\mathbb{P}^{1}$. We make the identification in such a manner that the $\operatorname{supp}(D)=\{0, \infty\}$, where in case $\left(2,1^{2}\right)$ we let $\infty$ be the image of the Weierstraß point. This identifies the stratum $\mathbb{P} \mathcal{H}^{\text {hyp }}\left(1^{4}\right), \mathbb{P} \mathcal{H}^{\text {hyp }}\left(2,1^{2}\right)$, $\mathbb{P H}^{\text {hyp }}(2,2)$ modulo the hyperelliptic involution with the configuration space of subsets of $\mathbb{C}^{\times}$of 8 resp. 7 resp. 6 elements, modulo the obvious $\mathbb{C}^{\times}$-action and, in the first and the last case, also modulo the involution. These are easily seen to be as asserted.

In order to understand how these loci lie in their strata, we need to have a unified picture that includes both non-hyperelliptic and hyperelliptic curves. While the anti-canonical model is adequate to discuss the case of a non-hyperelliptic curve, the bi-anti-canonical model is more suited to analyze what happens near the hyperelliptic locus. The degeneration of the del Pezzo surfaces using the anti-canonical model only was analyzed in [8].

\section{The bicanonical model}

In this section we show and investigate how a degeneration of a plane quartic into a hyperelliptic curve of genus 3 is obtained by putting its ambient projective plane in $\mathbb{P}^{5}$ via the Veronese embedding and let it then degenerate into a quartic cone. The underlying idea 
is borrowed from J. Shah's approach to the moduli space of K3 surfaces of degree 2 [9]. The same construction was also employed by Hassett [4] to understand limits of del Pezzo surfaces of degree 2 .

Given a curve $C$ of genus 3, then the canonical map

$$
k: C \rightarrow P_{C}:=\check{\mathbb{P}}\left(\mathrm{H}^{0}\left(\mathrm{C}, \omega_{\mathrm{C}}\right)\right)
$$

is an embedding in a projective plane unless $C$ is hyperelliptic, in which case it factors through the hyperelliptic involution (which we will always denote by $\tau$ ) and has a conic as image. On the other hand, the bicanonical map

$$
k^{\prime}: C \rightarrow \mathrm{P}_{\mathrm{C}}^{\prime}:=\check{\mathbb{P}}\left(\mathrm{H}^{0}\left(\mathrm{C}, \omega_{\mathrm{C}}^{\otimes 2}\right)\right)
$$

is always an embedding in a 5-dimensional projective space and this allows us to identify $\mathrm{C}$ with its image in $P_{C}^{\prime}$. Let

$$
j: \mathrm{P}_{\mathrm{C}} \hookrightarrow \check{\mathbb{P}}\left(\operatorname{Sym}^{2} \mathrm{H}^{0}\left(\mathrm{C}, \omega_{\mathrm{C}}\right)\right)
$$

be the Veronese embedding. The multiplication map

$$
m: \operatorname{Sym}^{2} \mathrm{H}^{0}\left(\mathrm{C}, \omega_{\mathrm{C}}\right) \rightarrow \mathrm{H}^{0}\left(\mathrm{C}, \omega_{\mathrm{C}}^{\otimes 2}\right)
$$

induces a rational map $\left[\mathrm{m}^{*}\right]: \mathrm{P}_{\mathrm{C}}^{\prime} \rightarrow \check{\mathbb{P}}\left(\operatorname{Sym}^{2} \mathrm{H}^{\mathrm{O}}\left(\mathrm{C}, \omega_{\mathrm{C}}\right)\right)$ and $\mathrm{C}$ will always lie on $\mathrm{V}_{\mathrm{C}}:=$ $\left[\mathrm{m}^{*}\right]^{-1} j\left(\mathrm{P}_{\mathrm{C}}\right)$. It is therefore of interest to determine what $V_{C}$ is like.

\subsection{Non-hyperelliptic case}

For $C$ non-hyperelliptic the map $m$ is an isomorphism. Hence so is $\left[\mathrm{m}^{*}\right]$ and this shows that $V_{C}$ is a Veronese surface and naturally isomorphic to $P_{C}$. We also find that this isomorphism takes $k(C)$ to $k^{\prime}(C)$. Thus, $k^{\prime}$ embeds $C$ in $P_{C}^{\prime}$ as the intersection of $V_{C}$ with a quadric: $k^{\prime}(C)$ appears as a divisor of $\mathcal{O}_{\mathrm{V}_{\mathrm{C}}}(2)$.

\subsection{Hyperelliptic case}

The situation is somewhat more complicated for $\mathrm{C}$ hyperelliptic. The hyperelliptic involution $\tau$ acts as -1 in $\mathrm{H}^{0}\left(C, \omega_{C}\right)$ and hence trivially in $\operatorname{Sym}^{2} \mathrm{H}^{0}\left(C, \omega_{C}\right)$. But it acts in $\mathrm{H}^{0}\left(C, \omega_{C}^{\otimes 2}\right)$ as reflection. The image of $\mathrm{m}$ is the full fixed point hyperplane of this reflection and so $\mathrm{m}$ has 1-dimensional kernel and cokernel. The kernel of $m$ is of course spanned by an element of $\operatorname{Sym}^{2} \mathrm{H}^{0}\left(C, \omega_{C}\right)$, which, when viewed as a quadratic form on $\mathrm{H}^{0}\left(C, \omega_{C}\right)^{*}$, defines the image of $k$ (a conic in $P_{C}$ ). And so the hyperplane $\mathrm{H}_{\infty} \subset \check{\mathbb{P}}\left(\operatorname{Sym}^{2} \mathrm{H}^{0}\left(C, \omega_{C}\right)\right)$ defined by this kernel has the property that $j k(C)=H_{\infty} \cap j\left(P_{C}\right)$. It also follows that the fixed point set of $\tau$ in $\mathrm{P}_{\mathrm{C}}^{\prime}$ consists of a hyperplane $\mathrm{H}$ and a singleton $\{v\}$, that $\left[\mathrm{m}^{*}\right]$ establishes an isomorphism $\mathrm{H} \cong$ $\mathrm{H}_{\infty}$ (we will therefore identify the two) and that $\left[\mathrm{m}^{*}\right]: \mathrm{P}_{\mathrm{C}}^{\prime} \rightarrow \check{\mathbb{P}}\left(\operatorname{Sym}^{2} \mathrm{H}^{0}\left(\mathrm{C}, \omega_{C}\right)\right.$ ) is the linear projection with center $v$ onto $H$ followed by the embedding $H \hookrightarrow \check{\mathbb{P}}\left(\operatorname{Sym}^{2} H^{0}\left(C, \omega_{C}\right)\right)$. This implies that $V_{C}$ is the cone with vertex $v$ and base the image of $j k(C) \subset H$. The image $k^{\prime}(C)$ lies in the smooth part of the cone $V_{C}$ and (again) appears as a divisor of $\mathcal{O}_{V_{C}}(2)$.

Notice that the involution $\tau$ acts on each ray of $V_{C}$ as the unique nontrivial involution that fixes its intersection with $\mathrm{H}$ and the vertex $v$. Moreover, the linear projection maps $k^{\prime}(C)$ onto as a double cover branched along an 8-element subset $B=k^{\prime}(C) \cap j k(C)$, whose two sheets are exchanged by $\tau$.

Incidentally, we remark that there are two distinct types of rays $R \subset V_{C}$ (Fig. 3): 


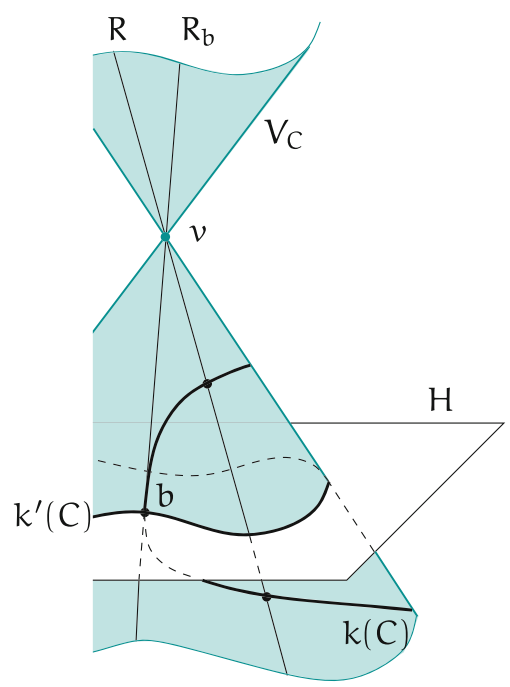

Fig. 3 The quartic cone $V_{C}$ for a hyperelliptic $C$

(a) an ordinary ray $\mathrm{R}$ : one that meets $\mathrm{k}^{\prime}(\mathrm{C})$ transversely in two points, so that $\tau$ acts nontrivially on $R \cap k^{\prime}(C)$;

(b) a Weierstraß ray $R=R_{b}$ : one that is tangent to $k^{\prime}(C)$ at a point $b \in B=k^{\prime}(C) \cap H_{\infty}$.

We also observe that the minimal resolution of the vertex of $V_{C}$ has as its exceptional set a rational curve of self-intersection -4 . The complement of the vertex in $V_{C}$ is isomorphic to the total space of the line bundle $\mathcal{O}_{\mathbb{P}^{1}}(4)$ over $\mathbb{P}^{1} \cong k(C)$. In other words, $V_{C}$ is obtained from a Segre-Hirzebruch surface $\Sigma_{4}$ by blowing down the (-4)-section. Thus, $V_{C}$ is a simply connected rational homology manifold with the homology of a complex projective plane and one can directly check that the restriction map $\mathrm{H}^{2}\left(\mathrm{P}_{\mathrm{C}}^{\prime} ; \mathbb{Z}\right) \rightarrow \mathrm{H}^{2}\left(\mathrm{~V}_{\mathrm{C}} ; \mathbb{Z}\right)$ is an isomorphism.

Indeed, the (-4)-singularity of the cone $V_{C}$ is homogeneous and $V_{C} \backslash H$ can be obtained as the quotient of an affine plane $\mathbb{A}^{2}$ by the diagonal action of $\mu_{4}$. The local Picard group of a (-4)-singularity is cyclic of order 4 with generator a ray $R$ and $4 R$ is a hyperplane section of $V_{C}$ and so locally principal. It follows that $\operatorname{Pic}\left(V_{C}\right)$ is generated by the class of $4 R$, and that since $(4 R) \cdot R=H \cdot R=1$, it follows that $R^{2}=1 / 4$. Clearly, $C \cdot R=2$.

Conversely, the pair $\left(V_{C}, C\right)$ is easily reconstructed up to isomorphism from an 8-element subset $B$ of a projective line $\mathbb{P}^{1}$, for the double cover of $\mathbb{P}^{1}$ ramified along B sits naturally in the total space of $\mathcal{O}_{\mathbb{P}^{1}}(4)$ and that total space can be identified with the complement of the vertex of a cone as above.

Notation In what follows, we will always identify a curve $C$ with its image through the bicanonical embedding. For a hyperelliptic $C$, we will write $\breve{C}$ for the rational curve $j k(C)$ sitting inside $\mathrm{V}_{\mathrm{C}}$.

\subsection{Near the hyperelliptic locus}

Let $\mathrm{C}_{0}$ be hyperelliptic of genus 3. It has a semi-universal deformation whose base is a smooth germ $(S, o)$ of dimension 6 with $T_{0} S$ naturally identified with the dual of $\mathrm{H}^{0}\left(C_{0}, \omega_{C_{0}}^{\otimes 2}\right)$. The universal property implies that the hyperelliptic involution $\tau$ extends to this universal deformation. The identification $\mathrm{T}_{\mathrm{O}} \mathrm{S} \cong \mathrm{H}^{0}\left(\mathrm{C}_{0}, \omega_{\mathrm{C}_{0}}^{\otimes 2}\right) *$ is $\tau$-equivariant. As we have seen $\tau$ 
acts as a reflection on $\mathrm{H}^{0}\left(\mathrm{C}_{0}, \omega_{\mathrm{C}_{0}}^{\otimes 2}\right)$ and so $\tau$ also acts as such on $\mathrm{T}_{\mathrm{o}} \mathrm{S}$. The action of $\tau$ on $(S, \mathrm{o})$ can be linearized in the sense that we can choose coordinates for $(S, 0)$ in terms of which $\tau$ is a reflection. The $(+1)$-eigenspace then parametrizes hyperelliptic deformations. But our interest concerns rather the $(-1)$-eigenspace, or more invariantly put, a transversal slice to the hyperelliptic locus invariant under $\tau$ (it is not unique). In other words, we consider a one-parameter deformation $f: \mathcal{C} \rightarrow \Delta$ of $C_{0}$ such that $C_{t}$ is non-hyperelliptic for $0 \neq t \in \Delta$, that the Kodaira-Spencer map is nonzero at $t=0$ and that the hyperelliptic involution $\tau$ extends to our family and takes $C_{t}$ to $C_{-t}$.

The above construction carries over in families and we obtain a factorization of the relative bicanonical embedding over $\Delta$

$$
\mathcal{C} / \Delta \hookrightarrow \mathcal{V} / \Delta \subset \mathcal{P}^{\prime} / \Delta
$$

through a surface $\mathcal{V}$ over $\Delta$ with central fiber $V_{0}$ a cone over a rational rational normal curve and general fiber a Veronese surface and we obtain $\mathcal{C}$ as divisor of a section $s$ of $\mathcal{O}_{\mathcal{V}}(2)$. We incidentally notice that $V_{0} \backslash H$ deforms to the complement of a hyperplane section of $V_{t}$, and so the Milnor fiber of this degeneration has the homotopy type of the complement of a conic in $\mathbb{P}^{2}$, that is, of a real projective plane.

We note that $\operatorname{Pic}(\mathcal{V}) \cong \operatorname{Pic}\left(V_{0}\right)$ is generated by $\mathcal{O}_{\mathcal{V}}(1)$. But on $V_{t}, t \neq 0, \mathcal{O}_{V_{t}}(1)$ represents twice the generator and so the image of the specialization map $\operatorname{Pic}\left(V_{0}\right) \cong \operatorname{Pic}(\mathcal{V}) \rightarrow \operatorname{Pic}\left(V_{t}\right)$ has index two. This corresponds (dually) to the fact that a line on the generic fiber of $\mathcal{V} / \Delta$ (that is, a line in $\left.\mathcal{P}\right|_{\Delta^{*}}$ under the image of Veronese map) may only extend as a Weil divisor with specialization to the sum of two rays (only four times a ray is a divisor of $\mathcal{O}_{V_{t}}(1)$ ). For example, each bitangent of $\left.\mathcal{C}\right|_{\Delta^{*}}$ specializes to the sum of two Weierstraß rays $R_{b}+R_{b^{\prime}}$ and this establishes a bijection between the 28 bitangents of $\left.\mathcal{C}\right|_{\Delta^{*}}$ and the collection of all 2-element subsets of B.

We deduce that the monodromy action of $\pi_{1}\left(\Delta^{*}\right)$ on the cohomology of the general fiber of $\mathcal{V} / \Delta$ is trivial. Indeed, the bundle $\mathcal{P}_{\Delta}^{\prime}$ is trivial and $H^{*}\left(P_{t}^{\prime} ; \mathbb{Q}\right) \rightarrow H^{*}\left(V_{t} ; \mathbb{Q}\right)$ is an isomorphism for all $t \in \Delta$. For essentially the same reason, the "half-monodromy" action $\tau: H^{*}\left(V_{t} ; \mathbb{Z}\right) \rightarrow H^{*}\left(V_{-t} ; \mathbb{Z}\right)$ is also trivial (after identifying $H^{*}\left(V_{-t} ; \mathbb{Z}\right)$ with $H^{*}\left(V_{t} ; \mathbb{Z}\right)$ via a path in $\left.\Delta^{*}\right)$.

\subsection{The double cover construction}

The action of $\tau$ on the family $\mathcal{C} / \Delta$ and so on the total space of the vector bundle $f_{*}\left(\omega_{f}^{\otimes 2}\right)$ induces an action on the total space of $\mathcal{O}_{\mathcal{V}}(1)$, which in particular fixes the fibers over $\breve{C}_{0}$. It acts as minus the identity on the fiber over $v$ and as plus the identity on its restriction to $\mathrm{H} \cap \mathrm{V}_{0}$. Hence, upon replacing $\mathrm{s}$ by $\mathrm{s}+\tau^{*} s$, we may assume that $s$ is $\tau$-invariant. As $s$ is a divisor for $\mathcal{C}$, we can construct the double cover $\pi: \mathcal{Y} \rightarrow \mathcal{V}$ branched over $\mathcal{C}$ inside the total space of $\mathcal{O}_{\mathcal{V}}(1)$ as the set of points whose square is a value of s. By construction it comes with an action of $\tau$. If we identify $\mathcal{V}$ with the zero section of this total space, then we see that $\mathcal{C}$ is also the ramification locus of $\pi$. We denote by เ the natural involution of $\mathcal{Y} / \mathcal{V}$. We remark that the actions of $\iota$ and $\tau$ commute.

For $t \neq 0, Y_{t}$ is clearly isomorphic to the del Pezzo surface $X_{C_{t}}$, whereas $Y_{0}$ has two isolated singular points $v^{+}, v^{-}$mapping to the vertex $v$ of $V_{0}$. Notice that besides the inclusion $C_{0} \subset \mathcal{C}$ there is another copy of $C_{0}$ embedded inside $Y_{0}$, namely preimage $\pi^{-1}\left(\breve{C}_{0}\right)$ and that the two meet each other in the 8 Weierstraß points. The involution $\mathrm{t}$ fixes $C_{0}$ pointwise and acts as the hyperelliptic involution on $\pi^{-1}\left(\breve{C}_{0}\right)$, whereas $\tau$ fixes $\pi^{-1}\left(\breve{C}_{0}\right)$ pointwise and acts nontrivially on $\mathrm{C}_{0}$.

Denote by $\tilde{R}$ be the preimage inside $Y_{0}$ of a ray $R \subset V_{0}$ (Fig. 4). 


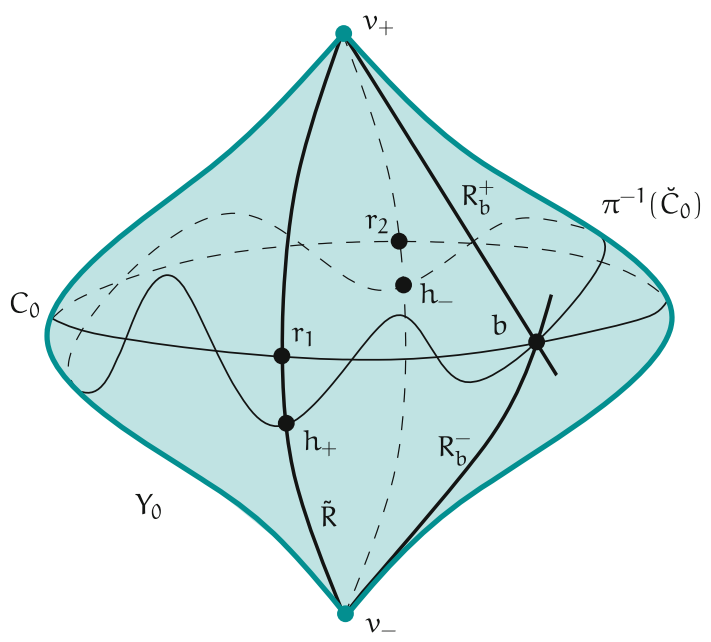

Fig. 4 The double cover $Y_{0}$ of the quartic cone $V_{0}$

(a) If $R$ is an ordinary ray, then $\tilde{R}$ is a smooth rational curve that doubly covers $R$. There are six special points on $\tilde{R}$ : the ramification points $r_{1}, r_{2}$, the singularities $v^{+}, v^{-}$of $Y_{0}$ and the preimages $h^{+}, h^{-}$of $R \cap H_{\infty}$. The involutions $\iota$ and $\tau$ on $\tilde{R}$ are characterized by the property that their fixed point pairs are $\left\{r_{1}, r_{2}\right\}$ and $\left\{h^{+}, h^{-}\right\}$respectively (so $\iota, \tau$ and $\iota \tau$ permute the 6 points respectively as $\left(v^{+} v^{-}\right)\left(h^{+} h^{-}\right),\left(v^{+} v^{-}\right)\left(r_{1} r_{2}\right)$ and $\left(h^{+} h^{-}\right)\left(r_{1} r_{2}\right)$.

(b) If $R_{b}$ is a Weierstraß ray through $b \in B$, then $\tilde{R}_{b}=R_{b}^{+} \cup R_{b}^{-}$, where $R_{b}^{ \pm}$is a smooth rational curve passing through $v^{ \pm}$and isomorphically mapping to $R_{b}$ and $R_{b}^{+} \cap R_{b}^{-}=\{b\}$ (b an ordinary double point of $\tilde{R}_{b}$ ). Both $\iota$ and $\tau$ act on $\tilde{R}_{b}$ by exchanging $v^{+}$and $v^{-}$and fixing $b$, and so exchanging $R_{b}^{+}$and $R_{b}^{-}$; but their action is different: in fact, $\iota \tau$ preserves each component and acts on $\mathrm{R}_{\mathrm{b}}^{ \pm}$as the only nontrivial involution that fixes $v^{ \pm}$and $\mathrm{b}$.

The variety $Y_{0}$ is a rational homology manifold too, and indeed the specialization map $\mathrm{H}^{*}\left(\mathrm{Y}_{0} ; \mathbb{Q}\right) \rightarrow \mathrm{H}^{*}\left(\mathrm{Y}_{\mathrm{t}} ; \mathbb{Q}\right)$ is an isomorphism, so that Poincaré duality with $\mathbb{Q}$ coefficients holds and all divisors are $\mathbb{Q}$-Cartier.

The intersection pairing of the liftings of Weierstraß rays satisfy $R_{b}^{+} \cdot R_{b}^{-}=1$ and $\left(R_{b}^{ \pm}\right)^{2}=$ $-3 / 4$ for every $b \in B$ and that for $b, b^{\prime} \in B$ distinct, $R_{b}^{+} \cdot R_{b^{\prime}}^{-}=0$ and $R_{b}^{ \pm} \cdot R_{b^{\prime}}^{ \pm}=1 / 4$. So $R_{b}^{ \pm}-R_{b^{\prime}}^{ \pm}$is a Cartier divisor of self-intersection -2 , whereas $R_{b}^{ \pm}+R_{b^{\prime}}^{ \pm}$is only a Weil divisor and has self-intersection -1 .

\subsection{Limits of exceptional classes}

As anticipated before, the 28 double tangents appear in the limit in the central fiber of $\mathcal{V}_{\Delta}$ as the $\left(\begin{array}{l}8 \\ 2\end{array}\right)$ couple of Weierstraß rays. The corresponding pairs of exceptional curves on the general fiber have a decent limit on the central fiber of $\mathcal{Y} \rightarrow \Delta$ and this limit can be described as follows. For every 2-element subset $\beta=\left\{b, b^{\prime}\right\} \subset B$, denote by $E_{\beta}^{+}$resp. by $E_{\beta}^{-}$the Weil divisor $\mathrm{R}_{\mathrm{b}}^{+}+\mathrm{R}_{\mathrm{b}^{\prime}}^{+}$resp. $\mathrm{R}_{\mathrm{b}}^{-}+\mathrm{R}_{\mathrm{b}^{\prime}}^{-}$inside $Y_{0}$. This describes the 56 limits of the exceptional curve on the general fiber: we get a flat family $\mathcal{E}_{\beta}^{ \pm} / \Delta$ whose general fiber is an exceptional curve on a del Pezzo surface of degree 2. Notice that the involutions ı and $\tau$ interchange $\mathcal{E}_{\beta}^{+}$and $\mathcal{E}_{\beta}^{-}$. 
The intersection numbers of these exceptional classes, insofar they are not self-intersections, take their values in $\{0,1,2\}$ and we have (compare [8], Lemma (6.4) for a different model):

(i) if $\beta \cap \beta^{\prime}$ is a singleton, then $E_{\beta, t}^{ \pm} \cdot E_{\beta^{\prime}, t}^{ \pm}=0$ and $E_{\beta, t}^{ \pm} \cdot E_{\beta^{\prime}, t}^{\mp}=1$,

(ii) if $\beta \cap \beta^{\prime}=\emptyset$, then $E_{\beta, t}^{ \pm} \cdot E_{\beta^{\prime}, t}^{ \pm}=1$ and $E_{\beta, t}^{ \pm} \cap E_{\beta^{\prime}, t}^{\mp}=0$ and

(iii) $E_{\beta, t}^{+} \cdot E_{\beta, t}^{-}=2$.

Since the Picard group of the general fiber of $\mathcal{Y} / \Delta$ is spanned by the exceptional classes, we see how that group specializes in the central fiber. We also see that on the central fiber, roots are still represented as differences of disjoint exceptional curves.

Notice that this construction leaves a trace on the relative Picard group of the generic fiber $\mathcal{Y}^{*} / \Delta^{*}$ (the superscript $*$ refers to restriction over $\Delta^{*}$ ), for it divides the exceptional curves of the generic fiber in two subsets that are interchanged by the involution $\mathrm{t}$. These are in fact separated by the character $\epsilon: \operatorname{Pic}\left(\mathcal{Y}^{*} / \Delta^{*}\right) \rightarrow\{ \pm 1\}$ which takes the value \pm 1 on $\mathcal{E}_{\beta}^{ \pm}$. In fact, we may identify $\operatorname{Pic}(\mathcal{Y} / \Delta)$ with the kernel of $\epsilon$.

We can be more explicit if $\mathcal{Y}^{*} / \Delta^{*}$ is given as a projective plane $\mathbb{P}_{\Delta^{*}}^{2}$ blown up in 7 numbered $\Delta^{*}$-valued points. This yields another basis of $\operatorname{Pic}\left(\mathcal{Y}^{*} / \Delta^{*}\right)$, namely $\ell, e_{1}, \ldots, e_{7}$, where $\ell$ is the preimage of the class of a line in $\mathbb{P}_{\Delta^{*}}^{2}$ and $e_{i}$ denotes the class of the exceptional divisor over the $i$-th point. Then $-K=3 \ell-\sum_{i=1}^{7} e_{i}$ is the anticanonical class and we can number the elements of $B: B=\left\{b_{0}, \ldots, b_{7}\right\}$ in such a manner that

$$
\begin{aligned}
e_{i}=\mathcal{E}_{\mathrm{b}_{0}, b_{i}}^{-}, & 1 \leq i \leq 7, \\
-\mathrm{K}-e_{i}=\mathcal{E}_{\mathrm{b}_{0}, b_{i}}^{+}, & 1 \leq i \leq 7, \\
\ell-e_{i}-e_{j}=\mathcal{E}_{\mathrm{b}_{i}, b_{j}}^{+}, & 1 \leq i<j \leq 7, \\
-\mathrm{K}-\left(\ell-e_{i}-e_{j}\right)=\mathcal{E}_{\mathrm{b}_{i}, b_{j}}^{-}, & 1 \leq i<j \leq 7 .
\end{aligned}
$$

The elements $\ell-e_{1}-e_{2}-e_{3}$ and $\left\{e_{i-1}-e_{i}\right\}_{i=2}^{7}$ make up a root basis of $\mathfrak{R}\left(\mathcal{Y}^{*} / \Delta^{*}\right)$. The roots in $\operatorname{ker}(\epsilon)$ are $e_{i}-e_{j}, 1 \leq i<j \leq 7$ and $\pm\left(-K-\ell+e_{i}\right)= \pm\left(2 \ell-e_{1} \cdots-\widehat{e_{i}} \cdots-e_{7}\right)$, $i=1, \ldots, 7$. This is in fact a root subsystem of type $\mathbf{A}_{7}$ for which $\left(-2 \ell+e_{2} \cdots+e_{7}, e_{1}-\right.$ $\left.e_{2}, \ldots, e_{6}-e_{7}\right)$ is a root basis.

We rephrase this for purposes of record in the following.

Proposition 4.1 Any root $\alpha \in \mathfrak{R}\left(\mathcal{Y}^{*} / \Delta^{*}\right)$ can be written either as a difference $\mathcal{E}_{\beta}^{ \pm}-\mathcal{E}_{\beta^{\prime}}^{ \pm}$ (the roots in $\operatorname{Ker}(\epsilon)$ ) or as $\mathcal{E}_{\beta}^{ \pm}-\mathcal{E}_{\beta^{\prime}}^{\mp}$ (the remaining ones), depending on whether $\beta \cap \beta^{\prime}$ is a singleton or empty. The roots of the first type are precisely the ones that lie in the image of $\operatorname{Pic}(\mathcal{Y} / \Delta)$ and make up a root subsystem $\mathfrak{R}_{0}(\mathcal{Y} / \Delta)$ of $\mathfrak{R}\left(\mathcal{Y}^{*} / \Delta^{*}\right)$ of type $\mathbf{A}_{7}$. Moreover, the permutation action of $\mathfrak{S}(\mathrm{B})$ on the collection of $\mathcal{E}_{\beta}^{ \pm}$'s defines an isomorphism of $\mathfrak{S}(\mathrm{B})$ onto the Weyl group of $\Re_{0}(\mathcal{Y} / \Delta)$ and identifies $\mathfrak{S}(\mathrm{B}) \times\langle\imath\rangle$ with the $\mathrm{W}\left(\mathcal{Y}^{*} / \Delta^{*}\right)$-stabilizer of $\operatorname{Ker}(\epsilon)$. Both $\tau$ and $\mathrm{l}$ act on $\mathfrak{R}\left(\mathcal{Y}^{*} / \Delta^{*}\right)$ as minus the identity.

Now let be given a family of conics $L_{t} \subset V_{t}$ degenerating into $L_{0}=R^{\prime}+R^{\prime \prime} \subset V_{0}$. There are three cases:

- $\mathrm{R}^{\prime}$ and $\mathrm{R}^{\prime \prime}$ are ordinary rays, accounting for $\mathbb{P} \mathcal{H}\left(1^{4}\right)_{\text {hyp }}$,

- $\mathrm{R}^{\prime}$ is ordinary and $\mathrm{R}^{\prime \prime}$ is Weierstraß, accounting for $\mathbb{P} \mathcal{H}\left(2,1^{2}\right)_{\text {hyp }}$,

- both $\mathrm{R}^{\prime}$ and $\mathrm{R}^{\prime \prime}$ are Weierstraß rays, accounting for $\mathbb{P} \mathcal{H}(2,2)_{\text {hyp }}$.

We think of $\mathcal{L}$ as defining a relative canonical divisor $\mathcal{D} / \Delta$ on the degenerating family $\mathcal{C} / \Delta$ and we need to understand how the basic invariant $\chi_{\mathcal{C}, \mathcal{D}}$ specializes over $0 \in \Delta$. This is the subject of the following two sections. 


\section{The open stratum}

This concerns the stratum $\mathbb{P} \mathcal{H}\left(1^{4}\right)$. We focus on the limiting behavior near the hyperelliptic locus and so we assume that we are in the situation of Sect. 4 and that $L_{0}$ is the sum of two ordinary rays $R^{\prime}$ and $R^{\prime \prime}$. Then the preimage of $L_{0}$ in $Y_{0}$ (denote it $\tilde{L}_{0}$ ) consists of two smooth rational components $\tilde{R}^{\prime}$ and $\tilde{R}^{\prime \prime}$ that meet at $v^{+}$and $v^{-}$. This is the central fiber of a genus one fibration $\tilde{\mathcal{L}} / \Delta$ with smooth general fiber: it is a degeneration of type $\mathrm{I}_{2}$; in particular, the central fiber is of multiplicative type. The $j$-function of such a degeneration has a pole of order two at $0 \in \Delta$. In fact, the involution $\tau$ nontrivially acts on the family $\tilde{\mathcal{L}}$ interchanging the fibers with the same $j$-invariant.

Lemma 5.1 Let $\alpha \in \mathfrak{R}(\mathcal{Y} / \Delta)$ be a root. Then $\chi_{\mathcal{C}, \mathcal{D}}(\alpha) \in \operatorname{Pic}(\tilde{\mathcal{L}} / \Delta)$ specializes as an element in the identity component of $\operatorname{Pic}\left(\tilde{\mathrm{L}}_{0}\right)$ if and only if $\alpha \in \mathfrak{R}_{0}(\mathcal{Y} / \Delta)$.

Proof Let $\hat{\mathcal{Y}}$ be obtained by blowing up $\mathcal{Y}$ at $v^{+}$and $v^{-}$. Then $\hat{Y}_{0}$ is the union of a double cover $\tilde{\Sigma}_{4}$ of a Segre-Hirzebruch surface and two copies $\Sigma_{0}^{ \pm}$of $\mathbb{P}^{1} \times \mathbb{P}^{1}$ glued along the two $(-4)$-sections $\sigma^{ \pm}$of $\tilde{\Sigma}_{4}$. Denote by $\widehat{R}^{\prime}, \widehat{R}^{\prime \prime}$ the strict transforms of $\tilde{R}^{\prime}, \tilde{R}^{\prime \prime}$ and by $\hat{\mathcal{L}}^{*}$ the preimage of $\tilde{\mathcal{L}}^{*}$ inside $\hat{\mathcal{Y}}^{*}$. Then its closure $\hat{\mathcal{L}}$ is a degeneration of type $\mathrm{I}_{4}$ as $\hat{\mathrm{L}}_{0}$ is the union of $\hat{R}^{\prime}, \hat{R}^{\prime \prime}, \sigma^{+}$and $\sigma^{-}$.

If $\widehat{R}_{b}^{ \pm}$is the strict transform of $R_{b}^{ \pm}$for some $b \in B$, then $\hat{R}_{b}^{ \pm}$meets $\hat{L}_{0}$ in the smooth locus of $\sigma^{ \pm}$. Hence, if $\hat{\mathcal{E}}_{\beta}^{ \pm} \subset \hat{\mathcal{Y}}$ denotes the strict transform of $\mathcal{E}_{\beta}^{ \pm}$, then the locus $\hat{\mathcal{L}} \cap \hat{\mathcal{E}}_{\beta}^{ \pm}$defines a relative divisor $\delta_{\beta}^{ \pm}$on $\hat{\mathcal{L}} / \Delta$ of degree 2 .

Let $\alpha \in \Re(\mathcal{Y} / \Delta)$ be represented by $\mathcal{E}_{\beta}^{ \pm}-\mathcal{E}_{\beta^{\prime}}^{ \pm}$resp. $\mathcal{E}_{\beta}^{ \pm}-\mathcal{E}_{\beta^{\prime}}^{\mp}$. Then $\chi \mathcal{C}, \mathcal{D}(\alpha) \in \operatorname{Pic}\left(\hat{\mathcal{L}}^{*} / \Delta^{*}\right)$ specializes to $\delta_{\beta}^{ \pm}(0)-\delta_{\beta^{\prime}}^{ \pm}(0)$ resp. $\delta_{\beta}^{ \pm}(0)-\delta_{\beta^{\prime}}^{\mp}(0)$ on $\hat{L}_{0}$. This specialization lies in the identity component of $\operatorname{Pic}\left(\hat{\mathrm{L}}_{0}\right)$ precisely if $\epsilon(\alpha)=1$. The conclusion follows because the pull-back induces an isomorphism between the identity components of $\operatorname{Pic}\left(\hat{\mathrm{L}}_{0}\right)$ and $\operatorname{Pic}\left(\tilde{\mathrm{L}}_{0}\right)$.

This describes in a rather concrete manner how the restriction homomorphism $\operatorname{Pic}\left(\mathcal{Y}^{*} / \Delta^{*}\right)$ $\rightarrow \operatorname{Pic}\left(\tilde{\mathcal{L}}^{*} / \Delta^{*}\right)$ specializes over the central fiber, for we also find that the limit $\chi_{\mathcal{C}, \mathcal{D}}(\alpha)$ exists precisely if $\alpha \in \mathfrak{R}_{0}(\mathcal{Y} / \Delta)$. Indeed, if $\epsilon(\alpha)=-1$, then after identifying $\operatorname{Pic}^{0}\left(\tilde{L}_{0}\right)$ with $\mathbb{C}^{\times}$, the value of $\chi_{\mathcal{C}, \mathcal{D}}(\alpha)$ tends to 0 or $\infty$ (depending on the identification). The involutions $\iota$ and $\tau$ act on $\operatorname{Pic}^{0}\left(\tilde{\mathrm{L}}_{0}\right)$ as the inversion and $\iota \circ \chi_{\mathcal{C}, \mathcal{D}}(\alpha)=\tau \circ \chi_{\mathcal{C}, \mathcal{D}}(\alpha)=\chi_{\mathcal{C}, \mathcal{D}}(-\alpha)$.

This gives rise to the following extension of $S\left(\mathbf{E}_{7}, \mathcal{C}_{1,1} / \mathcal{M}_{1,1}\right)$. We begin with what may be considered as a reconstruction of $\tilde{\mathcal{L}} / \Delta$. We start out with the algebraic torus $T=\left(\mathbb{C}^{\times}\right)^{2}$ and the automorphism $u$ of $T$ defined by $u\left(z_{1}, z_{2}\right)=\left(z_{1}, z_{1} z_{2}\right)$. This automorphism preserves the open subset $\mathcal{T}:=\Delta^{*} \times \mathbb{C}^{\times}$and generates a group $u^{\mathbb{Z}}$ thats acts properly and freely on $\mathcal{T}$. So the orbit space $\mathcal{F}^{*}$ is a complex manifold of dimension two. It maps homomorphically to $\Delta^{*}$ and this realizes $\mathcal{F}^{*}$ as the Tate curve over $\Delta^{*}$. We construct an extension $\overline{\mathcal{F}}$ of $\mathcal{F}^{*}$ over $\Delta$ by means of a familiar construction from toric geometry.

The coordinates give the lattice $\mathrm{N}_{\mathrm{T}}$ of one parameter subgroups of $\mathrm{T}$ a natural basis $\left(e_{1}, e_{2}\right)$. The rays in $N_{T} \otimes \mathbb{R}$ spanned by the vectors $e_{1}+n e_{2}$ with $n \in \mathbb{Z}$, and the sectors spanned by two successive rays define a partial polyhedral decomposition $\Sigma$ of $N_{T} \otimes \mathbb{R}$. This decomposition is clearly invariant under $u$. The associated torus embedding $T \subset T_{\Sigma}$ is a complex manifold of dimension two. Let $\mathcal{T}_{\Sigma} \supset \mathcal{T}$ be the interior of the closure of $\mathcal{T}$ in $T_{\Sigma}$. Then $u^{\mathbb{Z}}$ acts properly and freely on $\mathcal{T}_{\Sigma}$. We let $\overline{\mathcal{F}}$ be the orbit space of $\mathcal{T}_{\Sigma}$ with respect to the subgroup $\mathrm{u}^{2 \mathbb{Z}}$. This is also a complex manifold and it is the total space of a degeneration $\overline{\mathcal{F}} / \Delta$ of curves of genus 1 of type $\mathrm{I}_{2}$. We denote by $\mathrm{F}_{0}$ (resp. by $\mathcal{F}$ ) the complement of the two punctual strata in the central fiber inside $\overline{\mathrm{F}}_{0}$ (resp. inside $\overline{\mathcal{F}}$ ). The section $\sigma_{0}\left(z_{1}\right)=\left[z_{1}, 1\right]$ 
of $\mathcal{F} / \Delta$ makes it into a relative abelian variety, which we denote by $\mathcal{J} / \Delta$. We remark that $\mathrm{J}_{0} \cong \mathrm{Pic}^{0}\left(\mathrm{~F}_{0}\right) \times\{ \pm 1\} \cong \mathbb{C}^{\times} \times\{ \pm 1\}$.

The automorphism $u$ induces in $\overline{\mathcal{F}} / \Delta$ an order two translation. We have also natural commuting involutions $\iota$ and $\tau$ on $\overline{\mathcal{F}} / \Delta$, which are defined as $\iota\left[z_{1}, z_{2}\right]=\left[z_{1}, z_{2}^{-1}\right]$ and $\tau\left[z_{1}, z_{2}\right]=\left[-z_{1},-z_{2}^{-1}\right]$, so that the "half-monodromy" acts on $F_{t}$ as $\left[z_{2}\right] \mapsto\left[-z_{2}^{-1}\right]$.

The induced actions of $\iota$ and $\tau$ on $\mathcal{J} / \Delta$, given by $\iota\left[z_{1}, z_{2}\right]=\left[z_{1}, z_{2}^{-1}\right]$ and $\tau\left[z_{1}, z_{2}\right]=$ $\left[-z_{1}, z_{2}^{-1}\right]$, generate the automorphism group of $\mathcal{J} / \Delta$. We incidentally notice that the "halfmonodromy" acts on $J_{t}$ as the inverse (for the group operation on $J_{t}$ ).

If $\mathcal{J}(\Delta)$ denotes the group of sections of $\mathcal{J} / \Delta$, then we have a natural surjective homomorphism

$$
\mathrm{c}: \mathcal{J}(\Delta) \rightarrow \mathrm{J}_{0} \rightarrow\{ \pm 1\}
$$

The group of homomorphisms $\chi: \mathrm{Q}\left(\mathbf{E}_{7}\right) \rightarrow \mathcal{J}(\Delta)$ is represented by a $\Delta$-scheme that we shall denote by $\operatorname{Hom}\left(\mathrm{Q}\left(\mathbf{E}_{7}\right), \mathcal{J} / \Delta\right)$. Concretely, a basis $\alpha_{1}, \ldots, \alpha_{7}$ of $\mathrm{Q}\left(\mathbf{E}_{7}\right)$ identifies this $\Delta$-scheme with a sevenfold fiber product $\mathcal{J} \times_{\Delta} \mathcal{J} \times \cdots \times{ }_{\Delta} \mathcal{J}$. Its central fiber has $2^{7}$ connected components and these are canonically labeled by the group $\operatorname{Hom}\left(\mathrm{Q}\left(\mathbf{E}_{7}\right),\{ \pm 1\}\right)$. It follows from our discussion of the hyperelliptic limit that we must consider only some of these components, namely those that correspond to $\chi$ for which $c \chi: Q\left(\mathbf{E}_{7}\right) \rightarrow\{ \pm 1\}$ has as kernel a root sublattice of type $\mathbf{A}_{7}$. At this point we recall that the root subsystems of type $\mathbf{A}_{7}$ of $\mathbf{E}_{7}$ are transitively permuted by the Weyl group $W\left(\mathbf{E}_{7}\right)$ and that the sublattice spanned by such a subsystem has index 2 in $\mathrm{Q}\left(\mathbf{E}_{7}\right)$.

Let us denote by $\operatorname{Hom}_{\left(\mathbf{A}_{7}\right)}\left(\mathrm{Q}\left(\mathbf{E}_{7}\right), \mathcal{J} / \Delta\right)$ the locus in $\operatorname{Hom}\left(\mathrm{Q}\left(\mathbf{E}_{7}\right), \mathcal{J} / \Delta\right)$ defined by the $\chi$ with the above property. This subset is open and $W\left(\mathbf{E}_{7}\right)$-invariant. By the preceding remark, $W\left(\mathbf{E}_{7}\right)$ is transitive on the connected components of the central fiber, the stabilizer of a connected component being a Weyl group of type $\mathbf{A}_{7}$ times the center $\{ \pm 1\}$ of $W\left(\mathbf{E}_{7}\right)$. Removing the fixed point loci of reflections yields an open subset $\operatorname{Hom}_{\left(\mathbf{A}_{7}\right)}\left(\mathrm{Q}\left(\mathbf{E}_{7}\right), \mathcal{J} / \Delta\right)^{\circ}$ and we then put

$$
\mathrm{S}_{\left(\mathbf{A}_{7}\right)}\left(\mathbf{E}_{7}, \mathcal{J} / \Delta\right):=\operatorname{Aut}\left(\mathbf{E}_{7}, \mathcal{J} / \Delta\right) \backslash \operatorname{Hom}_{\left(\mathbf{A}_{7}\right)}\left(\mathrm{Q}\left(\mathbf{E}_{7}\right), \mathcal{J} / \Delta\right)^{\circ}
$$

If we fix a root subsystem of type $\mathbf{A}_{7}$ inside $\mathbf{E}_{7}$, then we see that the central fiber of $S_{\left(\mathbf{A}_{7}\right)}\left(\mathbf{E}_{7}, \mathcal{J} / \Delta\right)$ is identified with the component of $S\left(\mathbf{E}_{7}, J_{0}\right) \cong S\left(\mathbf{E}_{7}, \mathbb{C}^{\times} \times\{ \pm 1\}\right)$ that maps $\mathbf{A}_{7}$ to $\mathbb{C}^{\times} \times\{1\}$. Restriction to $\mathbf{A}_{7}$ identifies this in turn with $S\left(\mathbf{A}_{7}, \mathbb{C}^{\times}\right)$. We also observe that $S_{\left(\mathbf{A}_{7}\right)}\left(\mathbf{E}_{7}, \mathcal{J} / \Delta\right)$ maps to the quotient of $\Delta^{*}$ by the involution $z_{1} \mapsto-z_{1}$. So if we ignore the orbifold structure, then we have attached a copy of $S\left(\mathbf{A}_{7}, \mathbb{C}^{\times}\right)$to $S\left(\mathbf{E}_{7}, \mathcal{C}_{1,1} / \mathcal{M}_{1,1}\right)$. Now notice that the orbifold $S\left(\mathbf{A}_{7}, \mathbb{C}^{\times}\right)$is the moduli space of 8-element subsets of $\mathbb{C}^{\times}$given up a common scalar and up to a (common) inversion. This is also the moduli space of 10element subsets of a projective line endowed with a distinguished subset of 2 elements. If we pass to double covers ramifying over the remaining 8 points, we see that this is nothing but $\mathbb{P}\left(\mathcal{H}\left(1^{4}\right)_{\text {hyp }}\right)$. With this interpretation, the added locus is even identified with $\mathbb{P}\left(\mathcal{H}\left(1^{4}\right)_{\text {hyp }}\right)$ as an orbifold.

In order to make the construction global it is best to pass to a level two structure: consider the universal elliptic curve with a level two structure $\stackrel{\circ}{\mathcal{J}}^{[2]} / \mathcal{M}_{1,1}^{[2]}$ (a Deligne-Mumford stack). It comes with an $\operatorname{SL}(2, \mathbb{Z} / 2)$-action. We extend this to across the completed modular curve $\overline{\mathcal{J}}^{[2]} / \overline{\mathcal{M}}_{1,1}^{[2]}$ with curve of type $\mathrm{I}_{2}$ added as singular fibers. Denote by $\mathcal{J}^{[2]} / \overline{\mathcal{M}}_{1,1}^{[2]}$ the associated abelian stack and define the stack $\operatorname{Hom}_{\left(\mathbf{A}_{7}\right)}\left(\mathrm{Q}\left(\mathbf{E}_{7}\right), \mathcal{J}^{[2]} / \overline{\mathcal{M}}_{1,1}^{[2]}\right)$ in an evident 
manner. We then put

$$
\mathrm{S}_{\left(\mathbf{A}_{7}\right)}\left(\mathbf{E}_{7}, \mathcal{J} / \overline{\mathcal{M}}_{1,1}\right):=\operatorname{Aut}\left(\mathbf{E}_{7}, \mathcal{J}^{[2]} / \overline{\mathcal{M}}_{1,1}^{[2]}\right) \backslash \operatorname{Hom}_{\left(\mathbf{A}_{7}\right)}\left(\mathrm{Q}\left(\mathbf{E}_{7}\right), \mathcal{J}^{[2]} / \overline{\mathcal{M}}_{1,1}^{[2]}\right)^{\circ} .
$$

where $\operatorname{Aut}\left(\mathbf{E}_{7}, \mathcal{J}^{[2]} / \overline{\mathcal{M}}_{1,1}^{[2]}\right)=\mathrm{W}\left(\mathbf{E}_{7}\right) \times \operatorname{SL}(2, \mathbb{Z} / 2) /(-1,-1)$.

The right hand side contains $S\left(\mathbf{E}_{7}, \mathcal{J} / \overline{\mathcal{M}}_{1,1}\right)$ which fibers over $\mathcal{M}_{0,(4)} \subset \overline{\mathcal{M}}_{0,(4)}$, where the point added to $\overline{\mathcal{M}}_{0,(4)}$ (the cusp) is represented by a divisor on $\mathbb{P}^{1}$ which is twice a positive reduced divisor of degree two. Its complement is a $\mathbb{Z} / 2$-gerbe over $S\left(\mathbf{A}_{7}, \mathbb{C}^{\times}\right)$and we conclude:

Theorem 5.2 We have a natural isomorphism $\mathbb{P} \mathcal{H}\left(1^{4}\right) \cong \mathrm{S}_{\left(\mathbf{A}_{7}\right)}\left(\mathbf{E}_{7}, \mathcal{J} / \overline{\mathcal{M}}_{1,1}\right)$ over $\overline{\mathcal{M}}_{0,(4)}$ which identifies $\mathbb{P H}\left(1^{4}\right)_{\text {hyp }}$ with the fiber over the cusp (which, as we noted, has the structure of a $\mathbb{Z} / 2$-gerbe over $\mathrm{S}\left(\mathbf{A}_{7}, \mathbb{C}^{\times}\right)$).

This theorem gives us, at least in principle, access to the homotopy type of $\mathbb{P H}\left(1^{4}\right)$, although we admit that this may be hard in practice. A computation of its orbifold fundamental group looks feasible, however.

\section{The remaining strata}

\subsection{The stratum $\mathbb{P} \mathcal{H}\left(2,1^{2}\right)$}

We return to the limiting discussion in Sect. 4. But now we assume here that $L_{t}$ is tangent to $C_{t}$ at one point and $L_{t}$ limits to $L_{0}=R+R_{b_{0}}$, where $R$ is an ordinary ray and $R_{b_{0}}$ is a Weierstraß ray. The construction of the previous section now produces a family $\tilde{\mathcal{L}} / \Delta$ for which $\tilde{\mathcal{L}}^{*} / \Delta^{*}$ is a nodal curve of genus 1 , whose closed fiber $\tilde{L}_{0}$ has three components: $R_{b_{0}}^{ \pm}$ and $\tilde{R}$.

The exceptional curves meeting $\tilde{\mathrm{L}}_{0}$ at the singular point $v^{ \pm}$are $\mathcal{E}_{\mathrm{b}_{i}, \mathrm{~b}_{j}}^{ \pm}, 1 \leq i<j \leq 7$. The roots that are differences of two disjoint members taken from this collection make up a root subsystem $\mathfrak{R}_{0} \subset \mathfrak{R}$ of type $\mathbf{A}_{6}$ having $\left(e_{1}-e_{2}, \ldots, e_{6}-e_{7}\right)$ as a root basis. Moreover, the analogous of Lemma 5.1 holds, namely: if $\alpha \in \Re$, then $\chi_{\mathcal{C} / \mathcal{D}}(\alpha)$ specializes to an element of $\operatorname{Pic}^{0}\left(\tilde{\mathrm{L}}_{0}\right) \cong \mathbb{C}^{\times}$if and only if $\alpha \in \mathfrak{R}_{0}$.

This suggests the following construction (taken from [8]). Consider the torus $\operatorname{Hom}(Q(\Re)$, $\left.\mathbb{C}^{\times}\right)$. Its lattice of one parameter subgroups can be identified with the weight lattice $\operatorname{Hom}(Q(\Re), \mathbb{Z})$ and hence its tensor product with $\mathbb{R}$ with $\operatorname{Hom}(Q(\Re), \mathbb{R})$. The indivisible elements in $\operatorname{Hom}(Q(\Re), \mathbb{Z})$ whose kernel is root lattice of a subsystem of type $\mathbf{A}_{6}$ make up a $W(\mathfrak{R})$-orbit $\mathfrak{O}$ of a fundamental weight. Each of these elements spans an oriented ray in $\operatorname{Hom}(\mathrm{Q}(\mathfrak{R}), \mathbb{R})$ and the collection of such rays defines a toric extension

$$
\operatorname{Hom}\left(\mathrm{Q}(\Re), \mathbb{C}^{\times}\right) \subset \operatorname{Hom}_{\left(\mathbf{A}_{6}\right)}\left(\mathrm{Q}(\mathfrak{R}), \mathbb{C}^{\times}\right)
$$

To every subsystem $\mathfrak{R}_{0} \subset \mathfrak{R}$ of type $\mathbf{A}_{6}$ are associated two $\mathbb{R}_{+}$-rays and hence two copies of $\operatorname{Hom}\left(\mathrm{Q}\left(\mathfrak{R}_{0}\right), \mathbb{C}^{\times}\right)$. So if $\lambda \in \mathfrak{O}$ spans of one of the rays and if we let $\mathbb{C}^{\times}$act on $\mathbb{P}^{1}$ in the usual manner: $\zeta\left[z_{0}: z_{1}\right]=\left[\zeta z_{0}: z_{1}\right]$, then we can form $\mathbb{P}^{1} \times{ }_{\lambda} \operatorname{Hom}\left(Q(\Re), \mathbb{C}^{\times}\right)$ (which is isomorphic to $\left.\mathbb{P}^{1} \times\left(\mathbb{C}^{\times}\right)^{6}\right)$ and this glues the two copies of $\operatorname{Hom}\left(\mathrm{Q}\left(\mathfrak{R}_{0}\right), \mathbb{C}^{\times}\right)$on $\operatorname{Hom}\left(Q(\Re), \mathbb{C}^{\times}\right)$. Notice that $W(\Re)$ acts on $\operatorname{Hom}_{\left(\mathbf{A}_{6}\right)}\left(Q(\Re), \mathbb{C}^{\times}\right)$. The $W(\Re)$-stabilizer of the boundary torus defined by $\lambda \in \mathfrak{O}$ is the Weyl group of the $\mathbf{A}_{6}$-subsystem defined by $\lambda$. 
Let $\operatorname{Hom}_{\left(\mathbf{A}_{6}\right)}\left(\mathrm{Q}(\mathfrak{R}), \mathbb{C}^{\times}\right)^{\circ}$ be obtained by removing from $\operatorname{Hom}_{\left(\mathbf{A}_{6}\right)}\left(\mathrm{Q}(\mathfrak{R}), \mathbb{C}^{\times}\right)$the fixed point loci of the reflections in $W(\Re)$ and put

$$
\mathrm{S}_{\left(\mathbf{A}_{6}\right)}\left(\mathbf{E}_{7}, \mathbb{C}^{\times}\right):=\operatorname{Aut}\left(\mathbf{E}_{7}, \mathbb{C}^{\times}\right) \backslash \operatorname{Hom}_{\left(\mathbf{A}_{6}\right)}\left(\mathrm{Q}(\mathfrak{R}), \mathbb{C}^{\times}\right)^{\circ} .
$$

This contains $\mathrm{S}\left(\mathbf{E}_{7}, \mathbb{C}^{\times}\right)$as an open subset. Since the $\operatorname{Aut}\left(\mathbf{E}_{7}\right)=W\left(\mathbf{E}_{7}\right)$ and the $W\left(\mathbf{E}_{7}\right)$ stabilizer of a toric stratum in $\operatorname{Hom}_{\left(\mathbf{A}_{6}\right)}\left(\mathrm{Q}(\mathfrak{R}), \mathbb{C}^{\times}\right)$is a Weyl group of type $\mathbf{A}_{6}$, the added locus is isomorphic to a $\mathbb{Z} / 2$-gerbe over $W\left(\mathbf{A}_{6}\right) \backslash \operatorname{Hom}\left(\mathrm{Q}\left(\mathbf{A}_{6}\right), \mathbb{C}^{\times}\right)^{\circ}$. Arguing as before (see also [8]), we find

\section{Theorem 6.1 We have a natural isomorphism of orbifolds}

$$
\mathbb{P} \mathcal{H}\left(2,1^{2}\right) \cong \mathrm{S}_{\left(\mathbf{A}_{6}\right)}\left(\mathbf{E}_{7}, \mathbb{C}^{\times}\right)
$$

which extends the $\mathbb{Z} / 2$-gerbe $\mathbb{P H}\left(2,1^{2}\right)_{\text {hyp }} \rightarrow \mathrm{W}\left(\mathbf{A}_{6}\right) \backslash \operatorname{Hom}\left(\mathrm{Q}\left(\mathbf{A}_{6}\right), \mathbb{C}^{\times}\right)^{\circ}$ of Proposition 3.1.

\subsection{The stratum $\mathbb{P H}(2,2)$}

Here we need to deal with the case when $L_{t}$ tangent to $C_{t}$ in two points and $L_{t}$ limits to the union $L_{0}=R_{b_{0}}+R_{b_{7}}$ of two Weierstraß rays. Then $\tilde{\mathcal{L}} / \Delta$ is such that $\tilde{\mathcal{L}}^{*} / \Delta^{*}$ is s bigon curve and the closed fiber $\tilde{L}_{0}$ has four irreducible components: $R_{b_{0}}^{ \pm}$and $R_{b_{7}}^{ \pm}$. If $\mathcal{Y}^{*} / \Delta^{*}$ is given by blowing up 7 numbered points $p_{1}, \ldots, p_{7}$ in $\mathbb{P}_{\Delta^{*}}^{2}$ as before, then the root system $\mathfrak{R}^{\prime}:=$ $\mathfrak{R}\left(\mathcal{Y}^{*} / \Delta^{*}, \tilde{\mathcal{L}}^{*} / \Delta^{*}\right)$ is of type $\mathbf{E}_{6}$ and has root basis $\left(\ell-e_{1}-e_{2}-e_{3}, e_{1}-e_{2}, \ldots, e_{5}-e_{6}\right)$. Via the identification described in Sect. 4 we find that the exceptional curves through $v^{ \pm}$and without common components with $\tilde{\mathrm{L}}_{0}$ are $\mathcal{E}_{\mathrm{b}_{i}, \mathrm{~b}_{j}}^{ \pm}$with $1 \leq i<j \leq 6$. The roots that are differences of two disjoint members taken from this collection, and meeting both $v^{+}$or both $v^{-}$, make up a root subsystem $\mathfrak{R}_{0}^{\prime} \subset \mathfrak{R}^{\prime}$ of type $\mathbf{A}_{5}$ having $\left(e_{1}-e_{2}, \ldots, e_{5}-e_{6}\right)$ as root basis. If $\alpha \in \mathfrak{R}^{\prime}$, then $\chi_{\mathcal{C} / \mathcal{D}}(\alpha)$ specializes to an element of $\mathbb{C}^{\times}$if and only if $\alpha \in \mathfrak{R}_{0}^{\prime}$.

A construction similar to the one for the $\mathbb{P} \mathcal{H}\left(2,1^{2}\right)$-stratum then yields:

Theorem 6.2 We have a natural isomorphism of orbifolds

$$
\mathbb{P} \mathcal{H}(2,2) \cong \mathrm{S}_{\left(\mathbf{A}_{5}\right)}\left(\mathbf{E}_{6}, \mathbb{C}^{\times}\right)
$$

which extends the $\mathbb{Z} / 2$-gerbe $\mathbb{P} \mathcal{H}(2,2)_{\text {hyp }} \rightarrow \mathrm{S}\left(\mathbf{E}_{6}, \mathbb{C}^{\times}\right)$of Proposition 3.1.

\subsection{Orbifold fundamental groups}

The orbifold fundamental groups of an orbifold of the type $S_{\left(\Re_{0}\right)}\left(\mathfrak{R}, \mathbb{C}^{\times}\right)$, where $\mathfrak{R}$ is an irreducible and reduced root system and $\Re_{0} \subset \Re$ is a saturated root subsystem of corank one has essentially been determined in [8]. It is best described in terms of the extended (affine) root system, or rather, of the associated affine Coxeter system. We briefly recall the construction. Although much of what follows holds in greater generality, let us confine ourselves here to the case when $\Gamma$ is an affine Coxeter diagram of type $\hat{\mathbf{E}}_{7}$ (resp. $\hat{\mathbf{E}}_{6}$ ): this is T-shaped tree whose arms have edge length 3,3,1 (resp. 2,2,2). So the automorphism group $\operatorname{Aut}(\Gamma)$ of $\Gamma$ is a permutation group of a set of 2 (resp. 3 elements). Denote its vertex set by I. Then we have defined an associated Artin group $\operatorname{Art}_{\Gamma}$ given in terms of generators and relations: the generators are indexed by $I:\left\{t_{i}\right\}_{i \in I}$ and $t_{i}$ commutes with $t_{j}$ unless $i$ and $j$ span an edge of $\Gamma$ in which case we have a braid relation $t_{i} t_{j} t_{i}=t_{j} t_{i} t_{j}$. The group $\operatorname{Aut}(\Gamma)$ acts on $\operatorname{Art}_{\Gamma}$ by permuting its generators. 
To $\Gamma$ is associated a Coxeter group $W_{\Gamma}$ (the quotient of $\operatorname{Art}_{\Gamma}$ by putting $t_{i}^{2} \equiv 1$ for all $i \in I$ ) and a (Tits) representation of the Coxeter group on a real affine space $\mathbb{A}$ on which $W_{\Gamma}$ acts properly as an affine reflection group. The generating set I defines a fundamental simplex $\mathrm{K} \subset \mathbb{A}$. The group $\operatorname{Aut}(\Gamma)$ acts as a symmetry group on $\mathrm{K}$ and this action extends affine-linearly to $\mathbb{A}$. Thus $W_{\Gamma} \rtimes \operatorname{Aut}(\Gamma)$ (a quotient of $\operatorname{Art}_{\Gamma} \rtimes \operatorname{Aut}(\Gamma)$ ) acts on $\mathbb{A}$. Let $\mathbb{A}_{\mathbb{C}}^{\circ}$ denote the complexification of $\mathbb{A}$ with all its (affine) reflection hyperplanes removed. Then $\mathbb{A}_{\mathbb{C}}^{\circ}$ can be identified with a $W_{\Gamma} \rtimes \operatorname{Aut}(\Gamma)$-covering of $S\left(\Re, \mathbb{C}^{\times}\right)$and $\operatorname{Art}_{\Gamma} \rtimes \operatorname{Aut}(\Gamma)$ can be identified with the orbifold fundamental group of $S\left(\Re, \mathbb{C}^{\times}\right)$in such a manner that the covering projection $\mathbb{A}_{\mathbb{C}}^{\circ} \rightarrow S\left(\Re, \mathbb{C}^{\times}\right)$is given by the natural map $\operatorname{Art}_{\Gamma} \rtimes \operatorname{Aut}(\Gamma) \rightarrow W_{\Gamma} \rtimes \operatorname{Aut}(\Gamma)([8]$, Cor. 3.7).

The inclusion $S\left(\Re, \mathbb{C}^{\times}\right) \subset S_{\left(\Re_{0}\right)}\left(\Re, \mathbb{C}^{\times}\right)$induces a surjection on fundamental groups and essentially amounts to introducing one new relation: a loop around the added divisor gets killed in the fundamental group. The question is therefore how to represent that loop in $\operatorname{Art}_{\Gamma} \rtimes \operatorname{Aut}(\Gamma)$. This was addressed in [8] (Lemma $3.8 \mathrm{ff}$.). Let us describe this in some detail.

For every $i \in \mathrm{I}$, the subgraph $\Gamma_{i} \subset \Gamma$ obtained by removing $i$ and the edges connected to it is the graph of a finite Coxeter group $W_{\Gamma_{i}}$ which maps isomorphically onto the $W_{\Gamma}$-stabilizer of a vertex $v_{i}$ of $K$. The homomorphism $\operatorname{Art}_{\Gamma_{i}} \rightarrow \operatorname{Art}_{\Gamma}$ is known to be an embedding. We denote by $\Delta_{i}$ the image of the Garside element (see op. cit.) of Art $\Gamma_{i}$. Its image in $W_{\Gamma_{i}}$ is the longest element $w_{i}$ of $W_{\Gamma_{i}}$ and $w_{i}$ takes $K$ to a simplex opposite $v_{i}$. The opposition symmetry $s_{i}: \mathbb{A} \ni a \mapsto v_{i}-\left(a-v_{i}\right) \in \mathbb{A}$ with respect to $v_{i}$ composed with $w_{i}$ is represented by an automorphism of $\Gamma_{i}$ and $s_{i} w_{i}$ preserves $K$ if and only if this automorphism is the restriction of some $g_{i} \in \operatorname{Aut}(\Gamma)$. Let us call $i \in I$ quasi-special if that is the case. Then for a quasi-special $i \in I$ we have $g_{i} \Delta_{i}=\Delta_{i} g_{i}$ in $\operatorname{Art}_{\Gamma} \rtimes \operatorname{Aut}(\Gamma)$ and this element acts on $\mathbb{A}$ as $s_{i}$. If we have two distinct vertices $i, j \in I$ that are quasi-special, then $s_{j} s_{i}$ acts in $\mathbb{A}$ as translation over $2\left(v_{j}-v_{i}\right)$. Now $2\left(v_{j}-v_{i}\right)$ defines a one parameter subgroup $\gamma: \mathbb{C}^{\times} \rightarrow \operatorname{Hom}\left(\mathrm{Q}, \mathbb{C}^{\times}\right)$and under the identification of $\operatorname{Art}_{\Gamma} \rtimes \operatorname{Aut}(\Gamma)$ with the orbifold fundamental group of $S\left(\Re, \mathbb{C}^{\times}\right)$, the lift $\left(\Delta_{j} g_{j}\right)\left(\Delta_{i} g_{i}\right)^{-1}$ of $s_{j} s_{i}$ represents the conjugacy class of a simple loop in $S\left(\Re, \mathbb{C}^{\times}\right)$. This is the loop can be obtained by taking the $\gamma$-image of a circle $|z|=\varepsilon$ of small radius in $\operatorname{Hom}\left(\mathrm{Q}, \mathbb{C}^{\times}\right)$, applying a translate under the torus $\operatorname{Hom}\left(\mathrm{Q}, \mathbb{C}^{\times}\right)$so that the circle lies in $\operatorname{Hom}\left(\mathrm{Q}, \mathbb{C}^{\times}\right)^{\circ}$ and then mapping that circle to $S\left(\Re, \mathbb{C}^{\times}\right)$. We have $\left(\Delta_{j} g_{j}\right)\left(\Delta_{i} g_{i}\right)^{-1}=\Delta_{j} g_{j} g_{i}^{-1} \Delta_{i}^{-1}$ and so, if we have a toric extension in which such loops become contractible, then in this extension we have the relation $\Delta_{j}^{-1} \Delta_{i}=g_{j} g_{i}^{-1}$. Let us now treat the two cases separately.

Assume $\Gamma$ of type $\hat{\mathbf{E}}_{7}$. We take as quasi-special vertices one for which $\Gamma_{\mathrm{i}}$ is of type $\mathbf{E}_{7}$ (then the associated element of $\operatorname{Aut}(\Gamma)$ is the identity) and the unique one for which $\Gamma_{j}$ is of type $\mathbf{A}_{7}$ (then the associated $g \in \operatorname{Aut}(\Gamma)$ is not the identity). The loop in question is associated to the translation $v_{j}-v_{i}$ and so we are imposing the identity $\Delta_{\Gamma\left(\mathbf{A}_{7}\right)}^{-1} \Delta_{\Gamma\left(\mathbf{E}_{7}\right)}=$ g. Hence we can eliminate $g$ and we find:

Theorem 6.3 The orbifold fundamental group of $\mathbb{P H}\left(2,1^{2}\right)$ is the largest quotient of the affine Artin group $\mathrm{Art}_{\hat{\mathbf{E}}_{7}}$ for which $\Delta_{\Gamma\left(\mathbf{A}_{7}\right)}^{-1} \Delta_{\Gamma\left(\mathbf{E}_{7}\right)}$ is of order 2 and conjugation by $\Delta_{\Gamma\left(\mathbf{A}_{7}\right)}^{-1} \Delta_{\Gamma\left(\mathbf{E}_{7}\right)}$ permutes the generators (indexed by I) according to the nontrivial involution.

Assume now $\Gamma$ of type $\hat{\mathbf{E}}_{6}$. We let $i \in I$ be a terminal vertex (so that $\Gamma_{i}$ is of type $\mathbf{E}_{6}$ ) and let $j \in I$ be the unique vertex $\neq i$ connected with $i$ (so that $\Gamma_{j}$ is of type $\mathbf{A}_{5}+\mathbf{A}_{1}$ ). Both are quasi-special, define subgraphs $\Gamma\left(\mathbf{E}_{6}\right)$ and $\Gamma\left(\mathbf{A}_{5}+\mathbf{A}_{1}\right)$ (here the notation indicates the type) 
and define the same element of $\operatorname{Aut}(\Gamma)$ (namely the unique involution which fixes $i$ ). Then the loop in question is represented by $\Delta_{\Gamma\left(\mathbf{A}_{5}+\mathbf{A}_{1}\right)}^{-1} \Delta_{\Gamma\left(\mathbf{E}_{6}\right)}$ and hence:

Theorem 6.4 The orbifold fundamental group of $\mathbb{P H}(2,2)$ is the quotient of the semidirect product of the affine Artin group $\operatorname{Art}_{\hat{\mathbf{E}}_{6}}$ with the symmetry group $\operatorname{Aut}\left(\Gamma_{\hat{\mathbf{E}}_{6}}\right)$ of the $\hat{\mathbf{E}}_{6}$-graph defined by the relation $\Delta_{\Gamma\left(\mathbf{A}_{5}+\mathbf{A}_{1}\right)} \equiv \Delta_{\Gamma\left(\mathbf{E}_{6}\right)}$.

We may also write this as a semidirect product of $\operatorname{Aut}\left(\Gamma_{\hat{\mathbf{E}}_{6}}\right)$ and a quotient of $\operatorname{Art}_{\hat{\mathbf{E}}_{6}}$. The quotient is then obtained by imposing three such relations: one for every terminal vertex, so that $\operatorname{Aut}\left(\Gamma_{\hat{\mathbf{E}}_{6}}\right)$ still acts on it.

Needless to say that we don't know whether any of these has a contractible orbifold universal cover.

Acknowledgments E.L. wishes to thank the Mathematical Sciences Research Institute and the Tsinghua Mathematics Department for support and hospitality during the period part of this work was done. G.M. would like to thank Enrico Arbarello for frequent and useful exchange of ideas about spaces of abelian differentials and the Park City Mathematical Institute for hospitality in July 2011.

\section{References}

1. Allcock, D.: Completions, branched covers, Artin groups and singularity theory. Posted as arXiv: 1106.3459

2. Deligne, P.: Les immeubles des groupes de tresses généralisés. Invent. Math. 17, 273-302 (1972)

3. Eskin, A., Masur, H., Zorich, A.: Moduli spaces of abelian differentials: the principal boundary, counting problems, and the Siegel-Veech constants. Publ. Math. Inst. Hautes Études Sci. 97, 61-179 (2003)

4. Hassett, B.: Stable log surfaces and limits of quartic plane curves. Manuscripta Mathematica 100, 469-497 (1999)

5. Kontsevich, M., Zorich, A.: Lyapunov exponents and Hodge theory. Preprint IHES M/97/13 and posted as arXiv:hep-th/9701164v1

6. Kontsevich, M., Zorich, A.: Connected components of the moduli spaces of abelian differentials with prescribed singularities. Invent. Math. 153(3), 631-678 (2003)

7. Looijenga, E.: Cohomology of $\mathcal{M}_{\ni}$ and $\mathcal{M}_{3}^{1}$. In: Bödigheimer, C.-F., Hain, R.M. (eds.) Mapping Class Groups and Moduli spaces of Riemann Surfaces, Contemporary Mathematics, vol. 150, pp. 205-228. AMS, Providence, RI (1993)

8. Looijenga, E.: Artin groups and the fundamental groups of some moduli spaces. J. Topol. 1(1), 187-216 (2008)

9. Shah, J.: A complete moduli space for K3 surfaces of degree 2. Ann. Math. 112(3), 485-510 (1980) 